\title{
Modeling of Ion and Water Transport in the Biological Nanopore ClyA
}

\author{
Kherim Willems, ${ }^{\dagger, \ddagger}$ Dino Ruić, ${ }^{\boldsymbol{\top}, \ddagger}$ Florian Lucas, ${ }^{\S}$ Ujjal Barman, ${ }^{\ddagger}$ Johan \\ Hofkens, $^{\dagger}$ Giovanni Maglia, ${ }^{*, \S}$ and Pol Van Dorpe ${ }^{*, \dagger, \ddagger}$ \\ $\dagger K U$ Leuven, Department of Chemistry, Celestijnenlaan 200F, B-3001 Leuven, Belgium \\ $\$$ imec, Kapeldreef 75, B-3001 Leuven, Belgium \\ IKU Leuven, Department of Physics and Astronomy, Celestijnenlaan 200D, B-3001 \\ Leuven, Belgium \\ $\S$ University of Groningen, Groningen Biomolecular Sciences $\&$ Biotechnology Institute, \\ 9747 AG, Groningen, The Netherlands \\ E-mail: g.maglia@rug.nl; Pol.VanDorpe@imec.be
}

\begin{abstract}
In recent years, the protein nanopore cytolysin $\mathrm{A}$ (ClyA) has become a valuable tool for the detection, characterization and quantification of biomarkers, proteins and nucleic acids at the single-molecule level. Despite this extensive experimental utilization, a comprehensive computational study of ion and water transport through ClyA is currently lacking. Such a study yields a wealth of information on the electrolytic conditions inside the pore and on the scale the electrophoretic forces that drive molecular transport. To this end we have built a computationally efficient continuum model of ClyA which, together with an extended version of Poison-Nernst-Planck-Navier-Stokes (ePNP-NS) equations, faithfully reproduces its ionic conductance over a wide range of salt concentrations. These ePNP-NS equations aim to tackle the shortcomings of the traditional PNP-NS models by self-consistently taking into account the influence of both the ionic strength and the nanoscopic scale of the pore on all relevant electrolyte properties. In this study, we give both a detailed description of our ePNP-NS
\end{abstract}


model and apply it to the ClyA nanopore. This enabled us to gain a deeper insight into the influence of ionic strength and applied voltage on the ionic conductance through ClyA and a plethora of quantities difficult to assess experimentally. The latter includes the cation and anion concentrations inside the pore, the shape of the electrostatic potential landscape and the magnitude of the electro-osmotic flow. Our work shows that continuum models of biological nanopores - if the appropriate corrections are applied - can make both qualitatively and quantitatively meaningful predictions that could be valuable tool to aid in both the design and interpretation of nanopore experiments.

\section{Keywords}

biological nanopore; cytolysin A; continuum simulation; Poisson-Nernst-Planck and NavierStokes equations; single molecule

\section{Introduction}

The transport of ions and molecules through nanoscale geometries is a field of intense study that uses both experimental, theoretical and computational methods. ${ }^{1-6]}$ One of the primary driving forces behind this research is the development of nanopores as label-free, stochastic sensors at the ultimate analytical limit (i.e., single molecule). ${ }^{[10}$ Such detectors have applications ranging from the analysis of biopolymers such as DNA $\stackrel{11}{18}$ or proteins, $\frac{19}{23}$ to the detection and quantification of biomarkers, 24,30 to the fundamental study of chemical or enzymatic reactions at the single molecular level. 22131,34

Nanopores are typically operated in the resistive-pulse mode, where the fluctuations of their ionic conductance are monitored over time. ${ }^{79135}$ Experimentally, this is achieved by placing the nanopore between two electrolyte compartments and applying a constant DC (or AC) voltage across them. Due to the high resistance of the nanopore, virtually the full potential change occurs within (and around) the pore, resulting in a strong electric field $\left(10^{6}-10^{7} \mathrm{mV} \cdot \mathrm{nm}^{-1}\right)$ that electrophoretically drives ions and water molecules through 
it. $\underline{36}$ Hence, analyte molecules such as DNA or proteins are driven towards, and often through, the nanopore by a combination of Coulombic (electrophoretic) and hydrodynamic (electro-osmotic) forces. $\frac{36 \mid 40}{42}$ If successful, a translocation event is observed as a temporal fluctuation in the ionic conductance of the pore that serves as a unique molecular 'fingerprint' with which the molecule can be identified. $\stackrel{43}{ }$ Because the frequency, magnitude, duration and even noise levels of these events depend on the properties of both the analyte molecule and the nanopore itself, they are notoriously difficult to interpret unambiguously without a full understanding of the nanofluidic phenomena that underlie them.

The computational approaches most widely used to study nanofluidic transport in ion channels or biological nanopores comprise discrete methods such as molecular dynamics $(\mathrm{MD})^{44} \sqrt[51]{1}$ and Brownian dynamics (BD), $\underline{52}$ 58 and mean-field (continuum) methods based on solving the Poisson-Boltzmann (PB) equations 5960 and Poisson-Nernst-Planck (PNP) equations. $\frac{61-63}{63}$ The latter two can be coupled with the Navier-Stokes (NS) equation to include electro-osmotically or pressure driven fluid flow. $\stackrel{58 \mid 64}{ }$ Due to their explicit atomic or particle nature, MD and BD simulations are considered to yield the most accurate results. However, the large computational cost of simulating a complete biological nanopore system (100K-1M atoms) for hundreds of nanoseconds still necessitates the use of supercom-

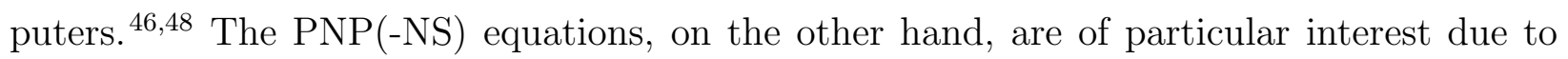
their low computational demands and analytical tractability. In a continuum approach, the simulated system is subdivided in several 'structureless' domains, the behavior of which is parameterized by material properties such as relative permittivity, diffusion coefficient, electrophoretic mobility, viscosity and density. Because these properties can only emerge from the collective behavior or interactions between small groups of atoms (i.e., the mean-field approximation), great care must be taken when using them to compute fluxes and fields at the nanoscale, where computational elements may only contain a few molecules. ${ }^{6566}$ Nevertheless, the PNP equations have been used extensively for the simulation of ion channels, $\frac{53 \mid 67 / 68}{5}$ biological nanopores ${ }^{58 / 63|69| 70}$ and their solid-state counterparts $39 \mid 71 / 73$ often with excellent 
qualitative, if not quantitative results..$[3[4] 6$

To remedy the shortcomings of PNP and NS theory, a number of modifications have been proposed over the years. These include, among others, 1) steric ion-ion interactions, 2) the effect of protein-ion/water interactions on their motility (i.e., diffusivity and electrophoretic mobility), 3) the concentration dependencies of ion motility, and solvent relative permittivity, viscosity and density.

The steric ion-ion interactions can be accounted for by computing the excess in chemical potential $\left(\mu_{i}^{\mathrm{ex}}\right)$ resulting from the finite size of the ions. $\frac{61|74| 75}{6 i l l e s p i e}$ et al. combined PNP and density functional theory — where $\mu_{i}^{\text {ex }}$ was split up in ideal, hard-sphere and electrostatic components - to successfully predict the selectivity and current of ion channels. ${ }^{62}$ In another approach, Kilić et al. derived a set of modified PNP equations based on the free energy functional of the Borukhov's modified PB model ${ }^{76}$ and observed significantly more realistic concentrations for high surface potentials compared to the classical PNP equations. ${ }^{77}$ To allow for non-identical ion sizes and more than two ion species, this model was later extended by $\mathrm{Lu}$ et al., who used it to probe the effect of finite ion size on the rate coefficients of enzymes. $\frac{78}{\underline{7}}$

The interaction of ions or small molecules such as water with the heavy atoms of proteins or DNA results in a strong reduction of their motility, as observed in MD simulations. $\frac{79[80}{.6}$ Since these effects happen only at distances $\leq 1 \mathrm{~nm}$, they can usually be neglected for macroscopic simulations. However, in small nanopores ( $\leq 10 \mathrm{~nm}$ radius), they comprise a significant fraction of the total nanopore radius and hence must be taken into account. $\underline{54 \mid 58 / 63 / 81}$ In continuum simulations, this can be achieved with the use pf positional-dependent ion diffusion coefficients. An example implementation is the 'soft-repulsion PNP' developed by Simakov and Kurnikova,, 63170 who used it to predict the ionic conductance of the $\alpha$ hemolysin nanopore. Similar reductions in ion diffusion coefficients have been proposed to improve PNP theory's estimations of the ionic conductance of ion channels. ${ }^{[67 \mid 68}$ The motility of water molecules is expressed by the NS equations as the fluid's viscosity. Hence, as 
also observed in MD simulations for water molecules near proteins $\frac{80}{}$ and confined in hydrophilic nanopores, $\underline{82}$ the water-solid interaction leads to a viscosity several times higher compared to the bulk values. Note that this is valid for hydrophilic interfaces only, as the lack of interaction with hydrophobic interfaces, such as carbon nanotubes, leads to a lower viscosity $\underline{85}$

It is well known that the self-diffusion coefficient $\mathcal{D}_{i}$ and electrophoretic mobility $\mu_{i}$ of an ion $i$ depends on the local concentrations of all the ions in the electrolyte. $\frac{86}{}$ Their values typically decrease with increasing salt concentration, and should not be treated as constants. Moreover, even though the well-known Nernst-Einstein (NE) relation $\mu_{i}=\mathcal{D}_{i} / k_{\mathrm{B}} T$ is strictly speaking only valid at infinite dilution and a good approximation at low concentrations $(<10 \mathrm{mM})$, it significantly overestimates the ionic mobility at higher salt concentrations. $\underline{86}, \underline{89}$ In an empirical approach, Baldessari and Santiago formulated an ionic-strength dependency of the ionic mobility based on the activity coefficient of the salt ${ }^{60}$ and showed excellent correspondence between the experimental and simulated ionic conductance of long nanochannels over a wide concentration range. $\underline{90}$ Alternatively, Burger et al. used a microscopic lattice-based model to derive a set of PNP equations with non-linear, ion density-dependent mobilities and diffusion coefficients that provided significantly more realistic results for ion channels. ${ }^{91}$ Note that other electrolyte properties, such as its viscosity, ${ }^{92}$ density $\stackrel{92}{\underline{92}}$ and relative permittivity, ${ }^{93}$ also significantly affect the ion and water flux. To better compute the charge flux in ion channels, Chen derived a new PNP framework ${ }^{94}$ that includes water-ion interactions in the form of a concentration-dependent relative permittivity and an additional ion-water interaction energy term.

To the best of our knowledge, no attempt has been made to consolidate all of the corrections discussed above into a single framework. Hence, we propose an extended set of PNP-NS (ePNP-NS) equations, which improves the predictive power of the PNP-NS equations at the nanoscale and beyond infinite dilution. Our ePNP-NS framework takes into account the finite size of the ions using a size-modified PNP theory, ${ }^{[78}$ and implements spatial-dependencies for 
the solvent viscosity, $\sqrt\left[80[84]{ } \text { the ion diffusion coefficients and their mobilities. }{ }^{[4] 79} \text { It also includes }\right.$ self-consistent concentration-dependent properties (based on empirical fits to experimental data) for both all ions in terms of diffusion coefficients and mobilities, $\underline{60187}$ and the solvent in terms of density, viscosity ${ }^{92}$ and relative permittivity $\underline{93}$. To validate our new framework, we applied it directly to a 2D-axisymmetric model of Cytolysin A (ClyA), a large protein nanopore that typically contains 12 subunits $^{\sqrt{95}}$ or more $e^{\sqrt{96}}$ and has been extensively used in experimental studies of both proteins $\sqrt{27 / 30 \mid 96} 100$ and DNA.17/101. This allowed us to gauge the qualitative and quantitative performance of the ePNP-NS equations and simultaneously elucidate previously unaccessible details about the environment inside the pore.

The remainder of this paper is organized as follows. In Mathematical model we describe the equations governing our ePNP-NS framework and detail the construction of the 2Daxisymmetric ClyA model. Next, in Results and Discussion, we validate our model by direct comparison of simulated ionic conductance with experimentally measured values. We then proceed to characterize the influence of the bulk ionic strength and the applied bias voltage on cation and anion concentrations inside the pore, the electrostatic potential distribution and magnitude of the electro-osmotic flow. Finally, we touch upon our key finding and their impact in Conclusions and describe our protocols in more detail in Materials and Methods.

\section{Mathematical model}

The use of continuum or mean-field representations for both the nanopore and the electrolyte enables us to efficiently compute the steady-state ion and water fluxes under almost any condition. The dynamic behavior of our complete system is described by the coupled Poisson, Nernst-Planck and Navier-Stokes (PNP-NS) equations, a well-known set of partial differential equations that describe the electrostatic field, the total ionic flux and the fluid flow, respectively. $\frac{6164 \mid 71}{}$ In this section we will describe all the components of the simulation, i.e., the 2D-axisymmetric nanopore geometry and the system of governing equations. 


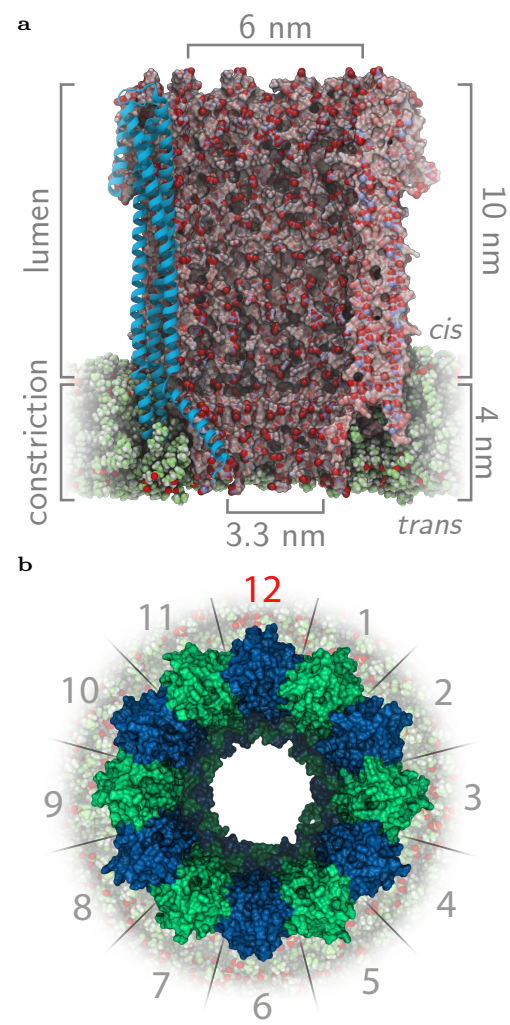

c
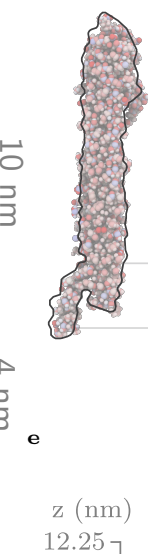

12.25

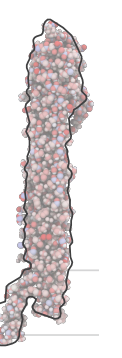

d

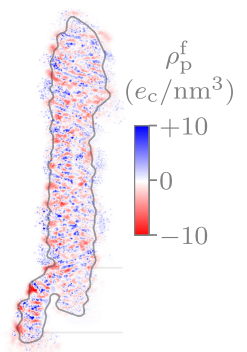

f

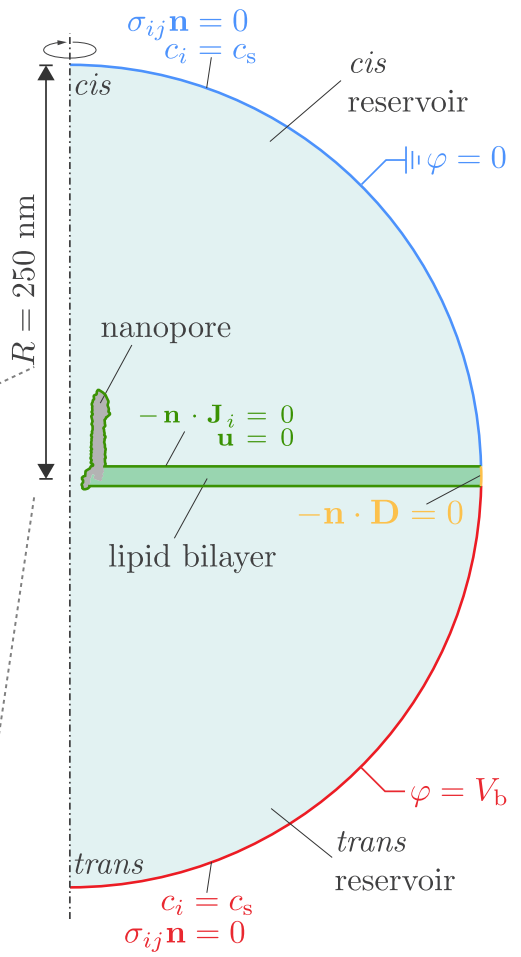

Figure 1. All-atom and 2D-axisymmetric models of ClyA. a Axial cross-sectional and b top views of the dodecameric nanopore ClyA-AS, 96 derived through homology modelling from the E. coli Cytolysin A crystal structure (PDBID: 2WCD ${ }^{95}$ ). Figures were rendered with VMD.102/103 (c) The 2D-axisymmetric geometry was derived directly from the all-atom model by computing the average inner and outer radii along the longitudinal axis of the pore, and hence closely follows the outline of a $30^{\circ}$ wedge out of the homology model. (d) The fixed space charge density ( $\left.\rho_{\text {pore }}^{f}\right)$ map of ClyA-AS, obtained by Gaussian projection of each atom's partial charge onto a 2D plane (see methods for details). (e+f) The 2D-axisymmetric simulation geometry of ClyA (grey) embedded in a lipid bilayer (green) and surrounded by a spherical water reservoir (blue). Note that all electrolyte parameters depend on the local average ion concentration $\langle c\rangle=\frac{1}{n} \sum_{i}^{n} c_{i}$ and that some are also influenced by the distance from the nanopore wall $d$. 


\section{Model geometry}

2D-axisymmetric model of ClyA ClyA is a relatively large protein nanopore that selfassembles on lipid bilayers to form $14 \mathrm{~nm}$ long hydrophilic channels. The interior of the pore can be divided into roughly two cylindrical compartments (Fig. 1a): the cis lumen $(\approx 6 \mathrm{~nm}$ diameter, $\approx 10 \mathrm{~nm}$ height), and the trans constriction ( $\approx 3.3 \mathrm{~nm}$ diameter, $\approx 4 \mathrm{~nm}$ height). Because ClyA consists of 12 identical subunits (Fig. 1b), it exhibits a high degree of radial symmetry, a geometrical feature that can be exploited to obtain meaningful results at a much lower computational cost. 58164171 However, this requires the reduction of the full $3 \mathrm{D}$ atomic structure and charge distribution to a realistic 2D-axisymmetric model. To this end, we constructed a full-atom homology model of ClyA-AS type I - a dodecameric variant of the wild type ClyA from S. Typhii artificially evolved for improved stability $\underline{96}$ and equilibrated it at $298.15 \mathrm{~K}$ for $30 \mathrm{~ns}$ in an explicit solvent with harmonic contraints on the protein backbone atoms (see Materials and Methods for details). From the final 5 ns of this trajectory we extracted 50 sets of atomic coordinates for ClyA (i.e., every $100 \mathrm{ps}$ ), in order to adequately represent the conformational diversity of the side chains. For each of these structures, we computed a 2D atomic density $\stackrel{104}{10}$ and charge ${ }^{46}$ map (see Materials and Methods for details), and the final maps were obtained through averaging. The geometry of the nanopore was then defined as the $25 \%$ contour line of the density map, which closely follows the outline of a $30^{\circ}$ 'wedge' of the full atom structure (Fig. 1c). The equilibrium charge map (Fig. 1d) was loaded directly into our solver as a linear interpolation function $\left(\rho_{\text {pore }}^{f}\right)$ and applied across all computational domains.

Global geometry. The complete system (Figs. 1e and 1f) consists of a large hemispherical electrolyte reservoir $(R=250 \mathrm{~nm})$, split through the middle into a cis and a trans compartment by a lipid bilayer $(h=2.8 \mathrm{~nm})$, which contains the nanopore at its center. Both the bilayer and the nanopore are represented by dielectric blocks (see Tab. 1 for parameters) that are impermeable to ions and water. 


\section{Governing equations}

In an attempt to improve upon the quantitative accuracy of the PNP-NS equations for nanopore simulations, we developed an extended version of these equations (ePNP-NS) and implemented it in the commercial finite element solver COMSOL Multiphysics (v5.4, www.comsol.com). Our ePNP-NS equations self-consistently take into account 1) the finite size of the ions, $\frac{76 / 78}{2)}$ the reduction of ion and water motility close to the nanopore walls, $\underline{5458 / 79|80| 83}$ and 3) the concentration dependency of ion diffusion coefficients and electrophoretic mobilities, as well as electrolyte viscosity, density and relative permittivity. $\underline{8792 \mid 93}$ Most of these corrections make use of using empirical functions that were fitted to experimental data (Tabs. 1 and $\mathrm{S} 2$ ).

Electrostatic field. As mentioned above, the electrostatic potential is evaluated using Poisson's equation

$$
\nabla \cdot\left(\varepsilon_{0} \varepsilon_{r} \nabla \varphi\right)=-\left(\rho_{\text {pore }}^{f}+\rho_{\text {ion }}\right)
$$

with $\varphi$ the electric potential, $\varepsilon_{0}$ the vacuum permittivity $\left(8.85419 \times 10^{-12} \mathrm{~F} \cdot \mathrm{m}^{-1}\right)$ and $\varepsilon_{r}$ local relative permittivity (Tab. S2).

The pore's fixed charge distribution, $\rho_{\text {pore }}^{f}$, (see Eq. 18) was derived directly from the full atom model of ClyA-AS. The ionic charge density in the fluid is given by

$$
\rho_{\text {ion }}=\mathcal{F} \sum_{i} z_{i} c_{i}
$$

with $\mathcal{F}$ Faraday's constant $\left(96485.33 \mathrm{C} \cdot \mathrm{mol}^{-1}\right)$, and $c_{i}$ the ion concentration and $z_{i}$ ion charge number of ion $i$.

To account for the concentration dependence of the electrolyte's relative permittivity, we 
used the expression

$$
\varepsilon_{r}(\langle c\rangle)=\varepsilon_{r}^{0} \varepsilon_{r}^{c}(\langle c\rangle)
$$

with $\langle c\rangle=\frac{1}{n} \sum_{i}^{n} c_{i}$ the average ion concentration, $\varepsilon_{r}^{0}$ the relative permittivity at infinite dilution and $\varepsilon_{r}^{c}(\langle c\rangle)$ a concentration dependent empirical function parameterized with experimental data.

Ionic flux. The total ionic flux $\boldsymbol{J}_{i}$ of each ion $i$ is given by the size-modified NernstPlanck equation, ${ }^{78}$ and can be expressed as the sum of diffusive, electrophoretic, convective and steric fluxes

$$
\boldsymbol{J}_{i}=-\left[\mathcal{D}_{i} \nabla c_{i}+z_{i} \mu_{i} c_{i} \nabla \varphi-\boldsymbol{u} c_{i}+\boldsymbol{\beta}_{\boldsymbol{i}} c_{i}\right]
$$

where

$$
\boldsymbol{\beta}_{\boldsymbol{i}}=\frac{a_{i}^{3} / a_{0}^{3} \sum_{j} a_{j}^{3} \nabla c_{j}}{1-\sum_{j} N_{\mathrm{A}} a_{j}^{3} c_{j}},
$$

and at steady state

$$
\frac{\delta c_{i}}{\delta t}=-\nabla \cdot \boldsymbol{J}_{i}=0
$$

with $\mathcal{D}_{i}$ the ion diffusion coefficient, $c_{i}$ the ion concentration, $z_{i}$ the ion charge number, $\mu_{i}$ the electrophoretic mobility of ion $i . \varphi$ is the electrostatic potential, $\boldsymbol{u}$ the fluid velocity and $N_{\mathrm{A}}$ Avogadro's constant $\left(6.022 \times 10^{23} \mathrm{~mol}^{-1}\right) . a_{i}$ and $a_{0}$ are steric cubic diameters of respectively ions and water molecules. Because currently there are no experimentally verified values available for $a_{i}$ and $a_{0}$, we set them to $0.5 \mathrm{~nm}(\max .13 .3 \mathrm{M})$ and $0.311 \mathrm{~nm}(\max .55 .2 \mathrm{M}) .74$ The reduction of the ionic motility at increasing salt concentrations and in proximity 
Table 1. Summary of the parameters and fitting equations used in the ePNP-NS equations.

\begin{tabular}{|c|c|c|c|}
\hline Property & Parameter $^{a}$ & Infinite dilution value ${ }^{b} /$ Fitting function $^{c}$ & Reference \\
\hline \multirow{4}{*}{ Relative permittivity } & $\varepsilon_{r, \mathrm{p}}$ & 20 & 104 \\
\hline & $\varepsilon_{r, \mathrm{~m}}$ & 3.2 & 105 \\
\hline & $\varepsilon_{r, \mathrm{f}}^{0}$ & 78.15 & 93 \\
\hline & $\varepsilon_{w}(\bar{c})$ & $1-\left(1-\frac{P_{1}}{P_{0}}\right) L\left(\frac{3 P_{2}}{P_{0}-P_{1}} \bar{c}\right)$ & 93 \\
\hline \multirow{4}{*}{$\begin{array}{l}\text { Ion self-diffusion } \\
\text { coefficient }\end{array}$} & $\mathcal{D}_{\mathrm{Na}^{+}}^{0}$ & $1.334 \times 10^{-9} \mathrm{~m}^{2} \cdot \mathrm{s}^{-1}$ & 87 \\
\hline & $\mathcal{D}_{\mathrm{Cl}^{-}}^{0}$ & $2.032 \times 10^{-9} \mathrm{~m}^{2} \cdot \mathrm{s}^{-1}$ & 87 \\
\hline & $\mathcal{D}_{i}^{c}(\bar{c})$ & $\left(1+P_{1} \bar{c}^{0.5}+P_{2} \bar{c}+P_{3} \bar{c}^{1.5}+P_{4} \bar{c}^{2}\right)^{-1}$ & This work \\
\hline & $\mathcal{D}_{i}^{w}(\bar{d})$ & $1-\exp \left(-P_{1}\left(\bar{d}+P_{2}\right)\right)$ & 6379 \\
\hline \multirow{4}{*}{$\begin{array}{l}\text { Ion electrophoretic } \\
\text { mobility }\end{array}$} & $\mu_{\mathrm{Na}^{+}}^{0}$ & $5.192 \times 10^{-4} \mathrm{~m}^{2} \cdot \mathrm{s}^{-1} \cdot \mathrm{V}^{-1}$ & 106 \\
\hline & $\mu_{\mathrm{Cl}^{-}}^{0}$ & $7.909 \times 10^{-4} \mathrm{~m}^{2} \cdot \mathrm{s}^{-1} \cdot \mathrm{V}^{-1}$ & 106 \\
\hline & $\mu_{i}^{c}(\bar{c})$ & $\left(1+P_{1} \bar{c}^{0.5}+P_{2} \bar{c}+P_{3} \bar{c}^{1.5}+P_{4} \bar{c}^{2}\right)^{-1}$ & This work \\
\hline & $\mu_{i}^{w}(\bar{d})$ & $1-\exp \left(-P_{1}\left(\bar{d}+P_{2}\right)\right)$ & 6379 \\
\hline \multirow{2}{*}{$\begin{array}{l}\text { Ion transport } \\
\text { number }\end{array}$} & $t_{\mathrm{Na}^{+}}^{0}$ & 0.396 & 106 \\
\hline & $t_{\mathrm{Na}^{+}}(\bar{c})$ & $\left(1+P_{1} \bar{c}^{0.5}+P_{2} \bar{c}+P_{3} \bar{c}^{1.5}+P_{4} \bar{c}^{2}\right)^{-1}$ & This work \\
\hline \multirow{3}{*}{ Dynamic viscosity } & $\eta^{0}$ & $8.904 \mathrm{~Pa} \cdot \mathrm{s}$ & 92 \\
\hline & $\eta^{c}(\bar{c})$ & $1+P_{1} \bar{c}^{0.5}+P_{2} \bar{c}+P_{3} \bar{c}^{2}+P_{4} \bar{c}^{3.5}$ & This work \\
\hline & $\eta^{w}(\bar{d})$ & $1+\exp \left(-P_{1}\left(\bar{d}-P_{2}\right)\right)$ & 80 \\
\hline \multirow{2}{*}{ Fluid density } & $\varrho^{0}$ & $997 \mathrm{~kg} \cdot \mathrm{m}^{-3}$ & 92 \\
\hline & $\varrho(\bar{c})$ & $1+P_{1} \bar{c}+P_{2} \bar{c}^{2}$ & This work \\
\hline
\end{tabular}

${ }^{a}$ Dependencies on either $\bar{c}=\langle c\rangle / 1 \mathrm{M}$ (dimensionless average ion concentration) and $\bar{d}=d / 1 \mathrm{~nm}$ (dimensionless distance from the nanopore wall); ${ }^{b}$ Values at infinite dilution for a system temperature of $291.15 \mathrm{~K} ;{ }^{c}$ These functions are empirical and hence have no physical meaning. The values of the fitting parameters $P_{x}$ of each property can be found in Tab. S2 and graphs of the fits in Fig. S1. 
to the nanopore walls was implemented self-consistently by replacing $\mathcal{D}_{i}$ and $\mu_{i}$ with the expressions

$$
\begin{gathered}
\mathcal{D}_{i}(\langle c\rangle, d)=\mathcal{D}_{i}^{0} \mathcal{D}_{i}^{c}(\langle c\rangle) \mathcal{D}_{i}^{w}(d) \\
\mu_{i}(\langle c\rangle, d)=\mu_{i}^{0} \mu_{i}^{c}(\langle c\rangle) \mu_{i}^{w}(d)
\end{gathered}
$$

where $\mathcal{D}_{i}^{0}$ and $\mu_{i}^{0}$ represent the values at infinite dilution. The concentration dependent factors $\mathcal{D}_{i}^{c}(\langle c\rangle)$ and $\mu_{i}^{c}(\langle c\rangle)$ are empirical functions fitted to experimental data (between 0 to $5 \mathrm{M} \mathrm{NaCl}$ ) of respectively the ion self-diffusion coefficients ${ }^{87}$ and the electrophoretic mobilities. ${ }^{106}\left[109\right.$ Likewise, the factors $\mathcal{D}_{i}^{w}(d)$ and $\mu_{i}^{w}(d)$ are empirical functions that introduce a spatial dependency on the distance from the nanopore wall $d$, and were parameterized by fitting to molecular dynamics data. $\underline{5463 / 79}$

Based on the observation that the diffusivity of nanometer- to micrometer-sized particles reduces significantly when confined in pores and slits of comparable dimensions,,$\underline{42110,113}$ Simakov et al. ${ }^{[6]}$ and Pederson et al. ${ }^{[58}$ reduced the ion motilities inside the pore as a function of the ratio between the ion and the nanopore radii. We chose not to include this correction into our model, as extrapolating its applicability for ions with a hydrodynamic radii comparable to size of the solvent molecules is questionable. 111114

Fluid flow. The fluid flow and pressure are given by the Navier-Stokes equations for incompressible fluids $\frac{115}{15}$

$$
(\boldsymbol{u} \cdot \nabla)(\varrho \boldsymbol{u})+\nabla \cdot \sigma_{i j}=\boldsymbol{F}
$$

where

$$
\sigma_{i j}=p \mathbf{I}-\eta\left[\nabla \boldsymbol{u}+(\nabla \boldsymbol{u})^{\top}\right]
$$


together with the continuity equations for the fluid density

$$
\boldsymbol{u} \cdot \nabla \varrho=0,
$$

and the fluid velocity

$$
(\boldsymbol{u} \cdot \nabla)(\varrho \boldsymbol{u}) \nabla \cdot(\varrho \boldsymbol{u})-\boldsymbol{u} \cdot \nabla \varrho=0
$$

with $\boldsymbol{u}$ the fluid velocity, $\varrho$ the fluid density, $\sigma_{i j}$ the hydrodynamic stress tensor, $\eta$ the viscosity and $p$ the pressure. The external body force density $\boldsymbol{F}$ that acts on the fluid is given by

$$
\boldsymbol{F}=e N_{\mathrm{A}} \rho_{\text {ion }} \boldsymbol{E}
$$

with $\boldsymbol{E}=-\nabla \varphi$ the electric field vector.

As with the previous equations, we introduced a concentration dependency and wall distance dependencies for $\eta$ and a concentration dependency for the $\varrho$ by replacing their constant values by

$$
\begin{aligned}
\eta(\langle c\rangle, d) & =\eta^{0} \eta^{c}(\langle c\rangle) \eta^{w}(d) \\
\varrho(\langle c\rangle) & =\varrho^{0} \varrho^{c}(\langle c\rangle)
\end{aligned}
$$

where $\eta^{0}$ and $\varrho^{0}$ are the values at infinite dilution (i.e., pure water). The empirical functions $\eta^{c}(\langle c\rangle), \varrho^{c}(\langle c\rangle)$ and $\eta^{w}(d)$ were parameterized via fitting to experimental ${ }^{[2]}$ and molecular dynamics $\sqrt[80]{ }$ data obtained from literature. 


\section{Boundary conditions}

The reservoir boundaries were set up, with Dirichlet conditions, to act as electrodes: the cis side was grounded $(\varphi=0)$ and a fixed, but changeable, bias potential was applied along the trans edge $\left(\varphi=V_{\mathrm{b}}\right)$. To simulate the presence of an endless reservoir, the ion concentration at both external boundaries were fixed to the bulk salt concentration $\left(c_{i}=c_{\mathrm{s}}\right)$ and the unconstrained flow in and out of the computational domain was enabled by means of a 'no normal stress' condition $\left(\sigma_{i j} \boldsymbol{n}=0\right)$. The boundary conditions on the edges of the reservoir shared with the nanopore and bilayer were set to no-flux $\left(-\boldsymbol{n} \cdot \boldsymbol{J}_{i}=0\right)$ and noslip $(\boldsymbol{u}=0)$, preventing the flux of ions through them and mimicking a sticky hydrophilic surface, respectively. Finally, a Neumann boundary condition was applied at the bilayer's external boundary $\left(-\boldsymbol{n} \cdot\left(\varepsilon_{0} \varepsilon_{r} \nabla \varphi\right)=0\right)$.

\section{Note on the concentration dependencies.}

All concentration dependent parameters use the local ionic strength rather than their individual ion concentrations. Though valid for electroneutral bulk solutions, this approximation no longer holds inside the electrical double layer (i.e., near charged surfaces or inside small nanopores), where local electroneutrality is violated. The main reasons for nevertheless making this simplification are the lack of non-bulk experimental data and the absence of a tractable analytical model. Furthermore, we will see that the current implementation of our concentration dependent functions will lead to an excellent agreement with the experimental data in all but the most extreme cases, justifying our choice a posteriori.

\section{PNP-NS vs. ePNP-NS}

The ePNP-NS equations revert into the regular PNP-NS equations disabling the steric flux $(\boldsymbol{\beta}=0)$ and by setting all concentration and wall distance functions to unity $\left(\varepsilon_{r}=\varepsilon_{r}^{0}\right.$, $\mathcal{D}_{i}=\mathcal{D}_{i}^{0}, \mu_{i}=\mu_{i}^{0}, \eta=\eta^{0}$ and $\left.\varrho=\varrho^{0}\right)$. 


\section{Results and Discussion}

The current-voltage (IV) relationships of many nanopores, ClyA included, often deviate significantly from Ohm's law. This is because the ionic flux arises from a complex interplay between the pore's geometry (e.g., size, shape and charge distribution), the properties of the surrounding electrolyte (e.g., salt concentration viscosity and relative permittivity) and the externally applied conditions (e.g., bias voltage, temperature and pressure). The ability of a computational model to quantitatively predict the ionic current of a nanopore over a wide range of bias voltages and salt concentrations strongly indicates that it captures the essential physics governing the nanofluidic transport. Hence, to validate our model, we experimentally measured the single channel ionic conductance of ClyA at a wide range of experimentally relevant salt concentrations and bias voltages. We compared these experimental data with the simulated ionic transport properties in terms of current, conductance, rectification and ion selectivity, of both the classical PNP-NS and the newly developed ePNP-NS equations. After validation, we proceed with describing the influence of the bias voltage $\left(V_{\mathrm{b}}\right)$ and bulk salt concentration $\left(c_{\mathrm{s}}\right)$ on the local ion and charge distribution and inside the pore. This is followed by a characterization of the electrostatic potential and the electrostatic energy landscape within ClyA for both cations and anions. We will conclude this section by discussing the properties of the electro-osmotic flow.

\section{Transport of Ions Through ClyA}

Ionic current and conductance. The ability of our model to reproduce the ionic current of a biological nanopore over a wide range of experimentally relevant conditions (between $V_{\mathrm{b}}=-150$ to $+150 \mathrm{mV}$ and for $c_{\mathrm{s}}=0.05,0.15,0.5,1$ and $3 \mathrm{M} \mathrm{NaCl}$ ) can be seen in Fig. 2a. Here, we compare IV relationships of ClyA-AS as measured experimentally ('expt.'), simulated using our 2D-axisymmetric model ('PNP-NS' and 'ePNP-NS') and naively analytically estimated ('bulk') using a resistor model of the pore ${ }^{96[116}$ (Eqs. S18 and S19]. Whereas the 
bioRxiv preprint doi: https://doi.org/10.1101/2020.01.08.897819; this version posted January 9, 2020. The copyright holder for this preprint (which was not certified by peer review) is the author/funder, who has granted bioRxiv a license to display the preprint in perpetuity. It is made available under aCC-BY 4.0 International license.

a
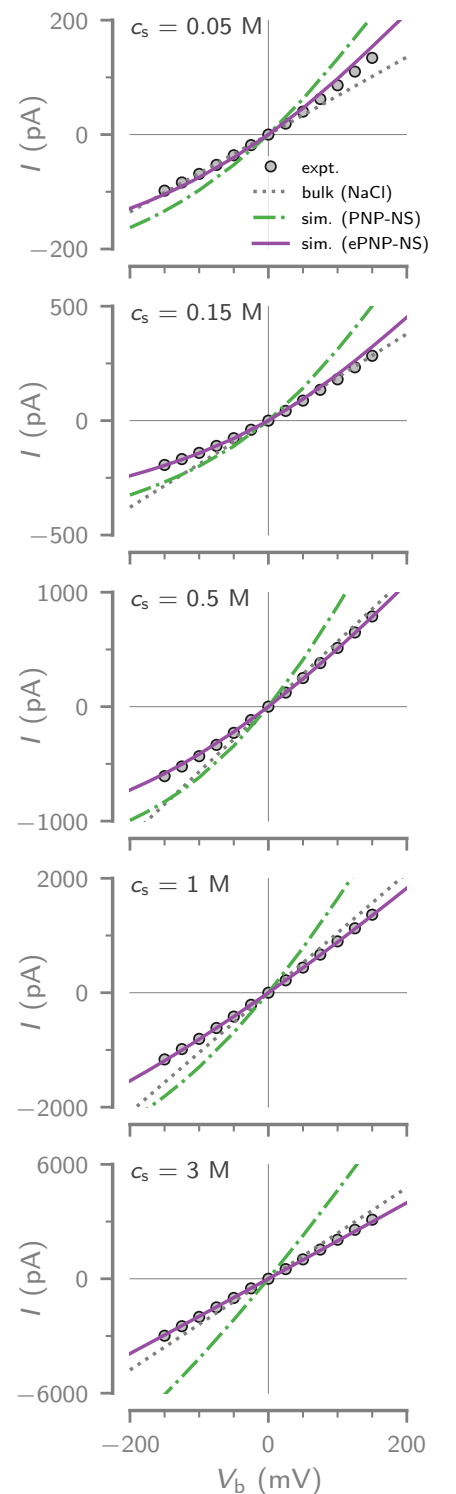
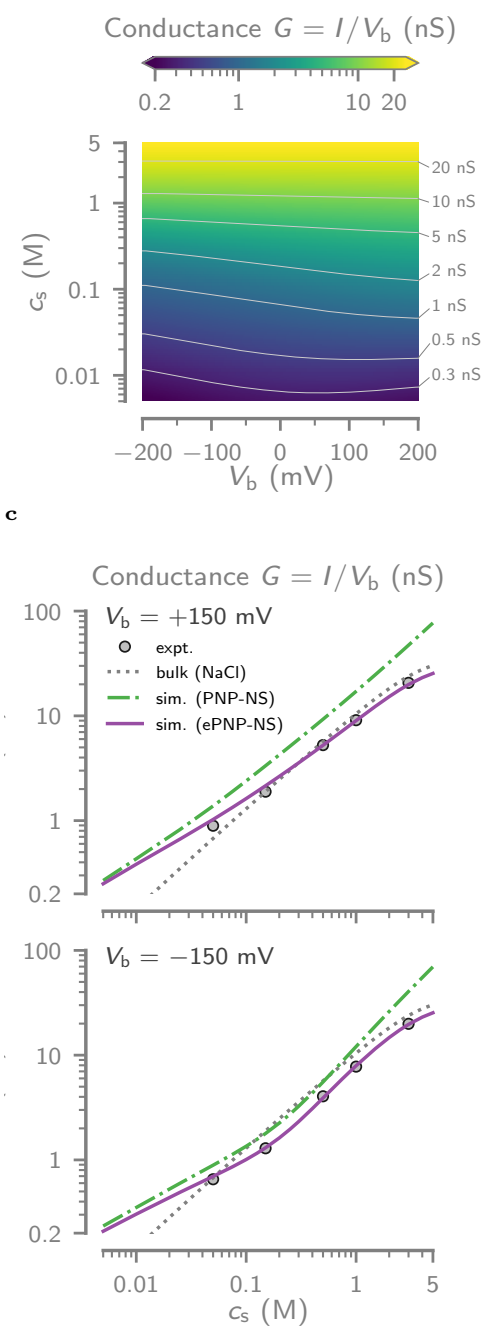
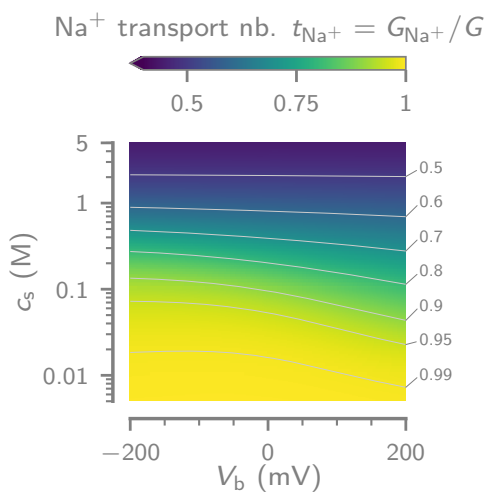

$\mathrm{Na}^{+}$transport nb. $t_{\mathrm{Na}^{+}}=G_{\mathrm{Na}^{+}} / \mathrm{G}$

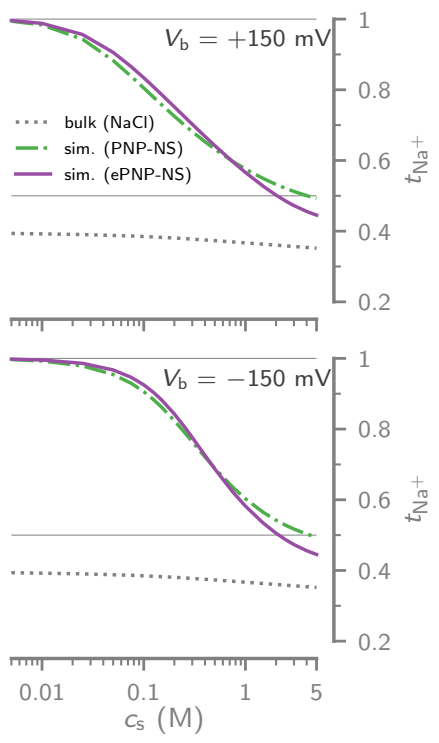

Figure 2. Measured and simulated ionic conductance and cation selectivity of single ClyA nanopores. a Comparison between the experimentally (expt.) measured, bulk pore model (bulk) and the simulated (PNP-NS and ePNP-NS) current-voltage (IV) curves of ClyA-AS at $25 \pm 1{ }^{\circ} \mathrm{C}$ between $V_{\mathrm{b}}=-200$ to $+200 \mathrm{mV}$, and for $c_{\mathrm{S}}=0.05,0.15,0.5,1$ and $3 \mathrm{M}$ $\mathrm{NaCl}$. The bulk current was calculated by Eq. S18 by modeling ClyA as two series resistors (Eq. S19, , 961116 using the bulk $\mathrm{NaCl}$ conductivity at the given concentrations. Experimental errors $(n=3)$ were smaller than the symbol size and hence not shown. (b) Contour plot of the simulated (ePNP-NS) ionic conductance $G=I / V_{\mathrm{b}}$ as a function of $V_{\mathrm{b}}$ and $c_{\mathrm{s}}$. (c) Log-log plots of $G$ as a function of $c_{\mathrm{s}}$ at $+150 \mathrm{mV}$ (top) and $-150 \mathrm{mV}$ (bottom) - comparing results obtained through experiments, PNP-NS and ePNP-NS simulations, and the simple resitor pore model. (d) Contour plot of the $\mathrm{Na}^{+}$transport number $t_{\mathrm{Na}^{+}}=G_{\mathrm{Na}^{+}} / G$, computed from the individual ionic conductances in the ePNP-NS simulation, as a function of $V_{\mathrm{b}}$ and $c_{\mathrm{S}}$. The $t_{\mathrm{Na}}+$ expresses the fraction of the ionic current is carried by $\mathrm{Na}^{+}$ions, i.e., the cation selectivity. e Simulated (PNP-NS and ePNP-NS) values of $t_{\mathrm{Na}}$ as a function of $c_{\mathrm{s}}$ for $+150 \mathrm{mV}$ (top) and $-150 \mathrm{mV}$ (bottom). Here, the 'bulk' line indicates the bulk $\mathrm{NaCl}$ cation transport number, represented by its empirical function $t_{\mathrm{Na}^{+}}\left(c_{\mathrm{s}}\right)$ (see Tab. 1 and Tab. S2. The solid grey line represents $t_{\mathrm{Na}}+=0.5$ 
classical PNP-NS equations consistently overestimated the ionic current, particularly at high salt concentrations, the predictions of the ePNP-NS equations corresponded closely to the measured values, especially at high ionic strengths $\left(c_{\mathrm{s}}>0.5 \mathrm{M}\right)$. The inability of the classical PNP-NS equations to correctly estimate the current is expected however, as in this regime the model parameters (e.g., diffusivity, mobility and viscosity, ...) begin to deviate significantly from their 'infinite dilution' values (see Fig. S1). At lower salt concentrations the ePNP-NS equations tended to minorly overestimate the ionic current, but the discrepancies were much smaller than those observed for PNP-NS. Finally, the bulk model managed to capture the currents suprisingly well at high salt concentrations and positive bias voltages, but faltered in the negative voltage regime.

The ability of a nanopore to conduct ions can be best expressed by its conductance: $G=I / V_{\mathrm{b}}$. We computed ClyA's conductance with the ePNP-NS equations as a function of bias voltage $\left(V_{\mathrm{b}}=-200\right.$ to $\left.+200 \mathrm{mV}\right)$ and bulk NaCl concentration $\left(c_{\mathrm{s}}=0.005\right.$ to $\left.5 \mathrm{M}\right)$, of which a contour plot can be found in Fig. 2b. The near horizontal contour lines in the upper part of the plot $\left(c_{\mathrm{s}}>1 \mathrm{M}\right)$ show that, at high ionic strengths, ClyA maintains the same conductance regardless of the applied bias voltage. This behavior changes at intermediate concentrations $\left(0.1 \mathrm{M}<c_{\mathrm{s}}<1 \mathrm{M}\right)$, where maintaining the same conductance level with increasing negative bias amplitudes requires increasing salt concentrations. Finally, at low salt concentrations $\left(c_{\mathrm{s}}<0.1 \mathrm{M}\right)$, the ionic conductance increases when reducing the negative voltage amplitude but subsequently levels out at positive bias voltages.

The cross-sections of the ionic conductance as a function of concentration at high positive and negative bias voltages (Fig. 2c), serve to demonstrate the differences between these respective regimes. The slope of the $\log -\log$ plot at $+150 \mathrm{mV}$ is highly linear, suggesting that only a single mechanism of ionic conduction is at work. As touched upon above, both the bulk model and the ePNP-NS equations manage to capture the conductance at high ionic strengths, while only the latter performs well at low ionic strengths. The predictions made with the PNP-NS equations overestimate the conductance over the entire concentration 
range, but they do converge at very low ionic strenghts. At $V_{\mathrm{b}}=-150 \mathrm{mV}$, the $\log -\log$ plot consists of two linear segments with a transition zone at $c_{\mathrm{s}} \approx 0.15 \mathrm{M}$. This behavior is captured qualitatively by both simulation methods, but a perfect quantitative match is only found for the ePNP-NS equations. The bulk model exhibits the same single-sloped trend as seen at $V_{\mathrm{b}}=+150 \mathrm{mV}$.

The difference in ionic conduction at opposing bias voltages is also known as ionic current rectification (ICR): $\alpha\left(V_{\mathrm{b}}\right)=G\left(+V_{\mathrm{b}}\right) / G\left(-V_{\mathrm{b}}\right)$. ICR is a phenomenon often observed in nanopores that are both charged, and contain a degree of geometrical asymmetry along the central axis of the pore. $5172[177$ As can be seen in Fig. S3, ClyA exhibits a strong degree of rectification, which is to be expected given its high negative charge $(-72 e$ at $\mathrm{pH} 7.5)$ and different $\operatorname{cis}(\approx 3.3 \mathrm{~nm})$ and trans $(\approx 6 \mathrm{~nm})$ entry diameters (Fig. 1a). We found $\alpha$ to increase monotoneously with the bias voltage magnitude, at least over the investigated range. We found the dependence of $\alpha$ on the ionic strength not to be monotonous, but rather rising rapidly to a peak value at $\approx 0.15 \mathrm{M}$, followed by a gradual decline towards unity at saturating salt concentrations.

The results and comparisons discussed above indicate that ClyA's conductivity is dominated by the bulk electrolyte conductivity above physiological salt concentrations $\left(c_{\mathrm{s}}>\right.$ $0.15 \mathrm{M})$. The breakdown of this simple dependency at lower ionic strengths is particularly evident at negative bias voltages and is likely caused by the overlapping of the electrical double layer inside the pore. This effectively excludes the co-ions $-\mathrm{Cl}^{-}$in the case-from the interior of the pore, preventing them from contributing to total ionic conductance and resulting in a conductance dominated by the surface charge. $\stackrel{118}{1}$ The presence of only a single ion type inside the pore may also offer an explanation as to why the ePNP-NS equations falter at low ionic strengths. Because our ionic mobilities are derived from bulk ionic conductances, i.e., for unconfined ions in a locally electroneutral environment, it is likely that our mobility model begins to break down under these conditions. $\frac{119}{11}$ Another cause of the discrepancies could be a change in the shape or diameter of the nanopore at low salt concentrations, which 
cannot be captured by our simulation due to the static nature of its geometry and charge distributions. Nevertheless, our simplified 2D-axisymmetric model, in conjunction with the ePNP-NS equations, is able to accurately predict the ionic current flowing through ClyA for a wide range of experimentally relevant ionic strengths and bias voltages. This suggests that we managed to capture the essential physical phenomena that drive the ion and water transport through the nanopore both qualitatively and quantitatively. Hence, we expect the distribution of the resulting properties (e.g., ion concentrations, ion fluxes, electric field and water velocity) to closely correspond to their real values.

Cation selectivity. The ion selectivity of a nanopore determines the preference with which it transports one ion type over the other. Experimentally, it is often determined by placing the pore in a salt gradient (i.e., different salt concentrations in the cis and trans reservoirs) and measuring the potential at which the nanopore current is zero (reversal potential, $\left.V_{\mathrm{r}}\right) .{ }^{96 / 101}$ The Goldman-Hodkin-Katz (GHK) equation can then be used to convert $V_{\mathrm{r}}$ into the permeability ratio $P_{\mathrm{Na}^{+}}=G_{\mathrm{Na}^{+}} / G_{\mathrm{Cl}^{-}}$. Here, we represent the ClyA's ion selectivity (Figs. 2d and 2e by the fraction of the total current that is carried by $\mathrm{Na}^{+}$ions: the apparent $\mathrm{Na}^{+}$ transport number $t_{\mathrm{Na}^{+}}=G_{\mathrm{Na}^{+}} /\left(G_{\mathrm{Na}^{+}}+G_{\mathrm{Cl}^{-}}\right)=P_{\mathrm{Na}^{+}} /\left(P_{\mathrm{Na}^{+}}+1\right)$.

Due its negatively charged interior, we found ClyA to be cation selective (i.e., $\left.t_{\mathrm{Na}^{+}}>0.5\right)$ for all investigated voltages up to a bulk salt concentration of $c_{\mathrm{s}} \approx 2 \mathrm{M} \mathrm{NaCl}(0.5$ contour line in Fig. 2d). Above this concentraion, $t_{\mathrm{Na}^{+}}$falls to a minimum of value of 0.45 at $c_{\mathrm{s}} \approx 5 \mathrm{M}$, which is still $\approx 1.27$ times its value in the bulk electrolyte $(0.35)$. This indicates that - even at saturation-ClyA remains preferential towards cations. Below $2 \mathrm{M}$, the ion selectivity increases logarithmically with decreasing salt concentrations, but it also becomes more sensitive to the direction and magnitude of the electric field: with negative bias voltages yield higher ion selectivities (Fig. 2e). For example, to reach a selectivity of $t_{\mathrm{Na}^{+}} \approx 0.9$, the salt concentration must fall to $0.05 \mathrm{M}$ at $+150 \mathrm{mV}$ and to only $0.125 \mathrm{M}$ at $-150 \mathrm{mV}$.

Using the reversal potential method, Franceschini et al. ${ }^{101}$ found ClyA's ion selectivity to 
be $t_{\mathrm{Na}^{+}}=0.66\left(P_{\mathrm{Na}^{+}}=1.9\right)$. In our ePNP-NS simultation, this corresponds the selectivity at $c_{\mathrm{s}}=0.5 \mathrm{M}\left(\right.$ at $\left.V_{\mathrm{b}}=0 \mathrm{mV}\right)$, a value that lies in between the cis $\left(1 \mathrm{M}, t_{\mathrm{Na}^{+}}=0.57\right.$, $\left.P_{\mathrm{Na}^{+}}=1.3\right)$ and trans $\left.\left(0.15 \mathrm{M}, t_{\mathrm{Na}^{+}}=0.84, P_{\mathrm{Na}^{+}}=5.4\right)\right)$ reservoir concentrations used in their experiment. Even though measuring the reversal potential can give valuable insights into the selectivity ion channels and small nanopores, it should be used with caution for larger nanopores such as ClyA. The GHK equation does not consider the ionic flux due to the electro-osmotic flow and assumes that the Nernst-Einstein relation holds for all used concentrations. These two effects should not be ignored as they contribute significantly to the nanopore's total conductance. Furthermore, because the ion selectivity depends strongly on the ionic strength, the measured reversal potential will necessarily be influenced by the chosen salt gradient and represent the selectivity at an undetermined intermediate concentration.

\section{Ion Concentration Distribution}

Following the validation of the model in preceding section, we now proceed by describing the local ionic concentrations inside ClyA. Detailed knowlegde of the ionic environment can be valuable to experimentalists who seek to trap and study single enzymes with ClyA. $\underline{30 \mid 97 / 100}$ Moreover, it gives insight into the origin of the ion current rectification, ion selectivity and the electro-osmotic flow. We evaluated the densities of both $\mathrm{Na}^{+}$and $\mathrm{Cl}^{-}$ions inside ClyA and investigated the effect of bulk salt concentration and the bias voltage on 1) the distribution of their concentration (Figs. 3a to 3c and 2) the accumulation of mobile charges inside the pore as a result of their asymmetric enhancement or depletion (Figs. $3 \mathrm{~d}$ to $3 \mathrm{f}$ ).

Relative cation and anion concentrations. The relative ion concentration averaged over the entire volume of the pore $\left(\left\langle c_{i} / c_{\mathrm{s}}\right\rangle_{\mathrm{p}}\right)$ as a function of bulk concentration (Fig. 3a), gives a good measure for global situation inside the pore. At low ionic strengths $\left(c_{\mathrm{s}}<0.05 \mathrm{M}\right)$, our simulation predicts a strong enhancement of the $\mathrm{Na}^{+}$(cation) concentration $\left(\left\langle c_{\mathrm{Na}^{+}} / c_{\mathrm{s}}\right\rangle_{\mathrm{p}}\right)$ and a clear depletion of the $\mathrm{Cl}^{-}$(anion) concentration $\left(\left\langle c_{\mathrm{Cl}^{-}} / c_{\mathrm{s}}\right\rangle_{\mathrm{p}}\right)$ inside the pore. This effect, 
a

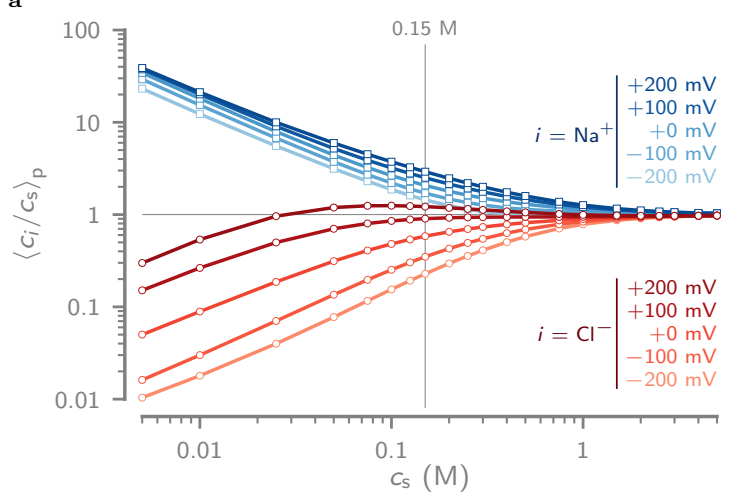

b
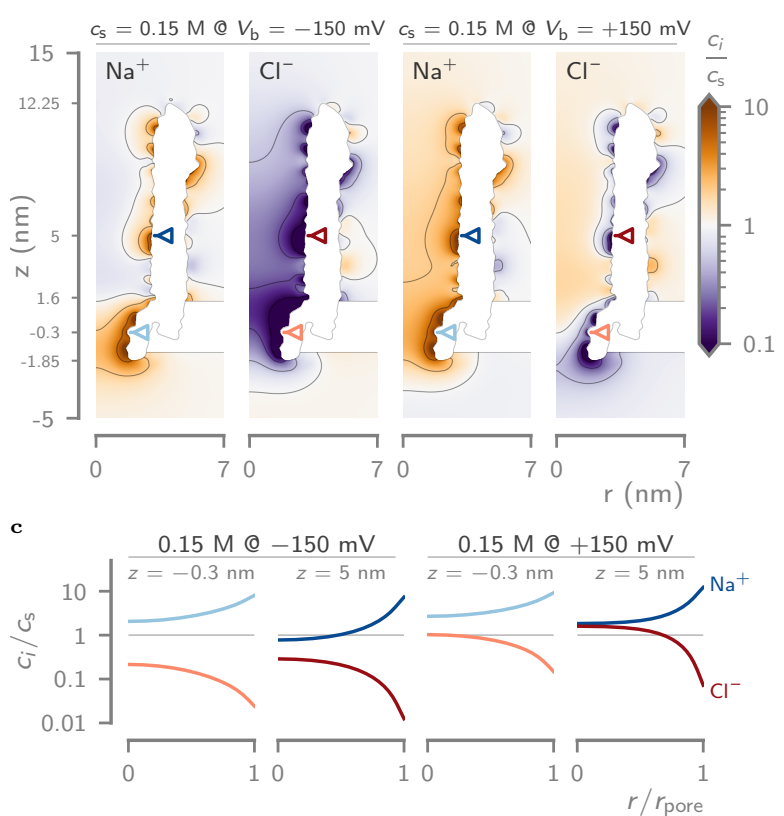

d
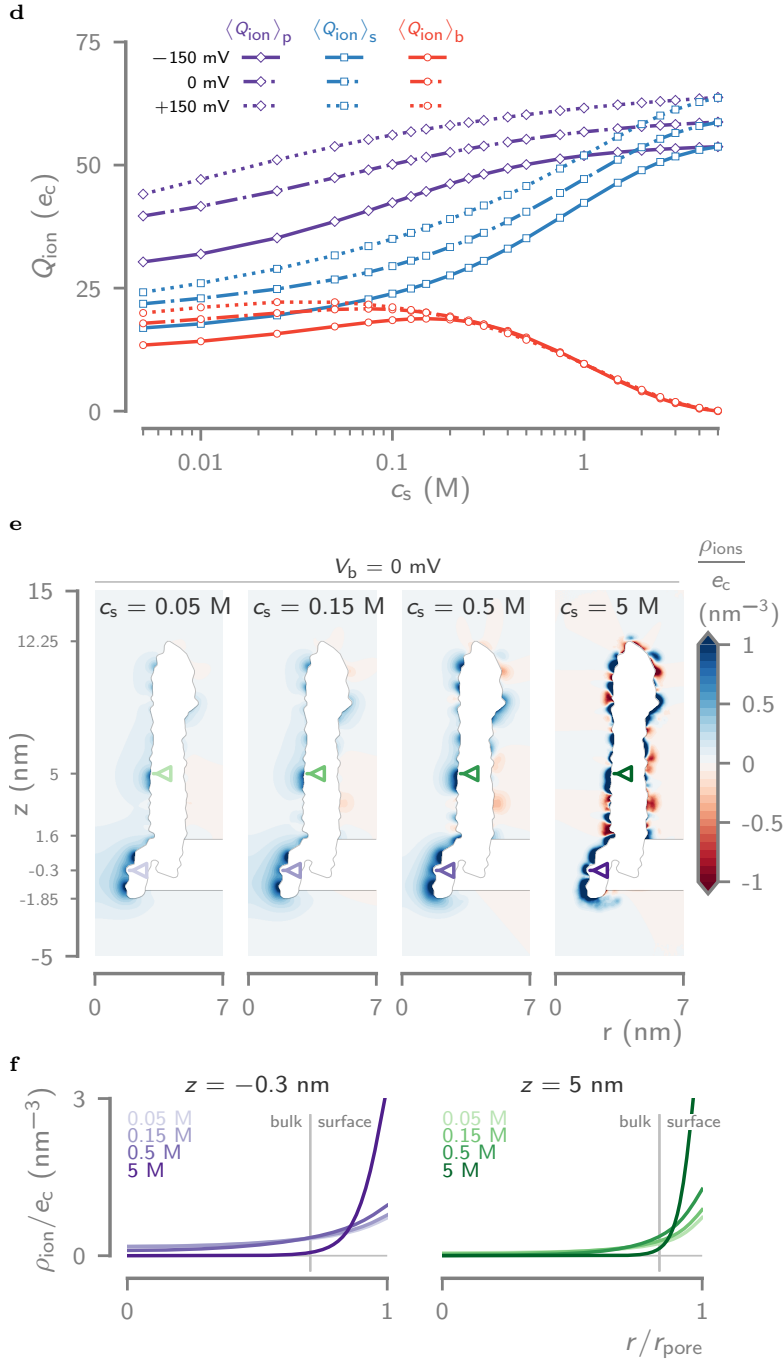

Figure 3. Ion concentration distribution inside ClyA. (a) Relative $\mathrm{Na}^{+}$and $\mathrm{Cl}^{-}$concentrations averaged over the entire pore volume $\left(\left\langle c_{i} / c_{\mathrm{s}}\right\rangle_{\mathrm{p}}\right)$ as a function of the reservoir salt concentration $\left(c_{\mathrm{s}}=0.005\right.$ to $\left.5 \mathrm{M}\right)$ and bias voltage $\left(V_{\mathrm{b}}=\right.$ -200 to $+200 \mathrm{mV}$ ). (b) Contour plots of the relative ion concentration $\left(c_{i} / c_{\mathrm{S}}\right)$ for both $\mathrm{Na}^{+}$and $\mathrm{Cl}^{-}$for $c_{\mathrm{S}}=0.15 \mathrm{M}$ and at $V_{\mathrm{b}}=-150$ and $+150 \mathrm{mV}$. (c) The relative $\mathrm{Na}^{+}$and $\mathrm{Cl}^{-}$concentration profiles along the radius of the pore, through the middle of the constriction $(z=-0.3 \mathrm{~nm})$ and the lumen $(z=5 \mathrm{~nm})$, as indicated by the arrows in (b). (d) The average number of ionic charges inside the pore $\left\langle Q_{\text {ion }}\right\rangle_{\mathrm{p}}$, is distributed between the those close to the pore's surface $\left\langle Q_{\text {ion }}\right\rangle_{\mathrm{s}}$, i.e., within $0.5 \mathrm{~nm}$ of the wall, and those in the 'bulk' of the pore's interior $\left\langle Q_{\text {ion }}\right\rangle_{\mathrm{b}}$. (e) Cross-section contour plots of the ion space charge density $\left(\rho_{\text {ion }}\right)$, expressed as number of elementary charges per $\mathrm{nm}^{3}$, at $V_{\mathrm{b}}=0 \mathrm{mV}$ and for $c_{\mathrm{s}}=0.05,0.15,0.5$ and $5 \mathrm{M}$. (f) Radial cross-sections of the $\rho_{\text {ion }}$ at the center of the constriction $(z=-0.3 \mathrm{~nm})$ and the lumen $(z=5 \mathrm{~nm})$ of ClyA. The vertical line represents the the division between ions in the 'bulk' $(d>0.5 \mathrm{~nm})$ of the pore and those located near its surface $(d \leq 0.5 \mathrm{~nm})$.

however, dimishes rapidly with increasing ionic strengths, which can be explained by the electrolytic screening of the negative charges lining the walls of ClyA (i.e., the electrical double layer). At low bulk concentrations the number of ions in the bulk is sparse, leading to the attraction and repulsion of respectively as many $\mathrm{Na}^{+}$and $\mathrm{Cl}^{-}$ions as the chemical potential allows. As the concentration increases, the overall availibility of ions improves 
and the extreme concentration differences between the pore and the bulk are no longer required to offset ClyA's fixed charges. For example, changing the bulk concentration from 0.005 to $0.05 \mathrm{M}$ under equilibrium conditions $\left(V_{\mathrm{b}}=0 \mathrm{mV}\right)$, causes the relative $\mathrm{Na}^{+}$and $\mathrm{Cl}^{-}$ concentrations to fall and rise from 34 to 4.4 and from 0.05 to 0.31 , respectively. At $c_{\mathrm{s}}=$ $0.15 \mathrm{M}$, the values still differ significantly from bulk $\left(\left\langle c_{\mathrm{Na}^{+}} / c_{\mathrm{s}}\right\rangle_{\mathrm{p}}=2.1\right.$ and $\left.\left\langle c_{\mathrm{Cl}^{-}} / c_{\mathrm{s}}\right\rangle_{\mathrm{p}}=0.58\right)$, but they fall back to $<15 \%$ of unity at $c_{\mathrm{s}}=1 \mathrm{M}\left(\left\langle c_{\mathrm{Na}^{+}} / c_{\mathrm{s}}\right\rangle_{\mathrm{p}}=1.14\right.$ and $\left.\left\langle c_{\mathrm{Cl}^{-}} / c_{\mathrm{s}}\right\rangle_{\mathrm{p}}=0.89\right)$.

We also observed a stark difference between their sensitivities to the applied bias voltage, particularly at low salt concentrations (Fig. 3a. left of $<0.15 \mathrm{M}$ line). Whereas the $\mathrm{Na}^{+}$ concentration shows only a limited response, the $\mathrm{Cl}^{-}$concentration changes much more dramatically. For example, at $c_{\mathrm{s}}=0.15 \mathrm{M}$ and from $V_{\mathrm{b}}=-150$ to $+150 \mathrm{mV},\left\langle c_{\mathrm{Na}^{+}} / c_{\mathrm{s}}\right\rangle_{\mathrm{p}}$ rises $\approx 1.7$-fold (1.6 to 2.7 ) and $\left\langle c_{\mathrm{Cl}^{-}} / c_{\mathrm{s}}\right\rangle_{\mathrm{p}}$ increases $\approx 3.8$-fold (0.28 to 1.06). This difference is clearly visualized by the contour plots of the relative ion concentrations $\left(c_{i} / c_{\mathrm{s}}\right)$ at $c_{\mathrm{s}}=0.15 \mathrm{M}$ and for $V_{\mathrm{b}}=$ text-150and $+150 \mathrm{mV}$ (Fig. 3b). They reveal that the trans constriction $(-1.85<z<1.6 \mathrm{~nm})$ remains depleted of $\mathrm{Cl}^{-}$and enhanced in $\mathrm{Na}^{+}$for both $V_{\mathrm{b}}=-150 \mathrm{mV}$ and $V_{\mathrm{b}}=+150 \mathrm{mV}$. This is not the case in the lumen $(1.6<z<12.25 \mathrm{~nm})$, in which the $\mathrm{Na}^{+}$concentration is bulk-like for $V_{\mathrm{b}}<0 \mathrm{mV}$ and enhanced for $V_{\mathrm{b}}>0 \mathrm{mV}$. Conversely, the number of $\mathrm{Cl}^{-}$ions becomes more and more depleted in the lumen for increasing negative bias magnitudes, and it is virtually bulk-like at higher positive bias voltages. This is further exemplified by the radial profiles of the ion concentrations (Fig. 3c) through the middle of the constriction $(z=-0.3 \mathrm{~nm})$ and the lumen $(z=5 \mathrm{~nm})$, which also clearly shows the extent of the electrical double layer.

Ion Charge Density. The formation of an electrical double layer inside the pore, and the resulting asymmetry in the cation and anion concentrations, gives rise to a net charge density inside the pore ( $\rho_{\text {ion }}$, Eq. 2). To investigate the distribution of these charges within ClyA, we divided the interior volume of the pore into a 'surface' and a 'bulk' region. The bulk region encompasses a cylindrical volume at the center of the pore up until $0.5 \mathrm{~nm}$ distance from the 
wall $(d \geq 0.5 \mathrm{~nm})$, and the surface region includes the remaining volume between the bulk and the nanopore wall $(d<0.5 \mathrm{~nm})$. Integration of $\rho_{\text {ion }}$ over the 'surface' and 'bulk' regions yields the average number of mobile charges present inside those locations (Fig. $3 \mathrm{~d}):\left\langle Q_{\text {ion }}\right\rangle_{\mathrm{b}}$ and $\left\langle Q_{\text {ion }}\right\rangle_{\mathrm{s}}$, respectively. Although the total number of charges inside the pore, $\left\langle Q_{\text {ion }}\right\rangle_{\mathrm{p}}=$ $\left\langle Q_{\text {ion }}\right\rangle_{\mathrm{b}}+\left\langle Q_{\text {ion }}\right\rangle_{\mathrm{s}}$, rises appreciatively with increasing reservoir concentrations, the majority of these additional charges are confined to the surface of the pore. Up until $c_{\mathrm{s}}=\approx 0.15 \mathrm{M}$, $\left\langle Q_{\text {ion }}\right\rangle_{\mathrm{p}}$ is distributed equally between the surface $(\approx+27 e)$ and bulk $(\approx+22 e)$ layers. At higher concentrations, the number of charges in the surface layer more than doubles (towards $\left\langle Q_{\text {ion }}\right\rangle_{\mathrm{s}}=+58 e$ at $5 \mathrm{M}$ ), and those in the bulk of the pore diminish (towards $\left\langle Q_{\text {ion }}\right\rangle_{\mathrm{b}} \approx 0 e$ at $5 \mathrm{M})$. The total number of mobile charges $\left\langle Q_{\text {ion }}\right\rangle_{\mathrm{p}}$ also depend on the applied bias voltage. As can be seen from our simulation results for three different voltages (Fig. 3d), $\left\langle Q_{\text {ion }}\right\rangle_{\mathrm{p}}$ is approximately +10 to +15 e higher at $V_{\mathrm{b}}=+150 \mathrm{mV}$ as compared to $V_{\mathrm{b}}=-150 \mathrm{mV}$ for the full range of ion concentrations. Interestingly, at higher salt concentrations this increased number of charges with voltage is fully accounted for by the surface charges, as the charge density in the bulk does not change with the applied voltage.

The cross-section contour plots of $\rho_{\text {ion }}$ inside ClyA for four different bulk concentrations $\left(c_{\mathrm{s}}=0.05,0.15,0.5\right.$ and $\left.5 \mathrm{M}\right)$ reveal the redistribution of the mobile charges with increasing ionic strength in more detail. Up until a bulk concentration of $c_{\mathrm{s}}=\leq 0.5 \mathrm{M}$, the electrical double layer inside the pore overlaps significantly with itself (Fig. 3e, 3 leftmost panels), giving rise to a net positive charge spread diffusively throughout the entire pore. Moreover, the absence of $\mathrm{Cl}^{-}$ions prevents the formation of any significant negative charge densities next to the few positively charged residues lining the pore walls. The situation at high salt concentrations (e.g., $5 \mathrm{M})$ is very different, with almost no charge density within the 'bulk' of the pore lumen $(d \geq 0.5 \mathrm{~nm})$, but with pockets of highly charged and alternating positive and negative charge densities close to the nanopore wall (Fig. 3e, rightmost panel). This sharp confinement is shown clearly by the radial density profiles (Fig. 3f) drawn through the constriction ( $z=-0.3 \mathrm{~nm}$, purple triangles) and the lumen ( $z=5 \mathrm{~nm}$, green triangles). 
a

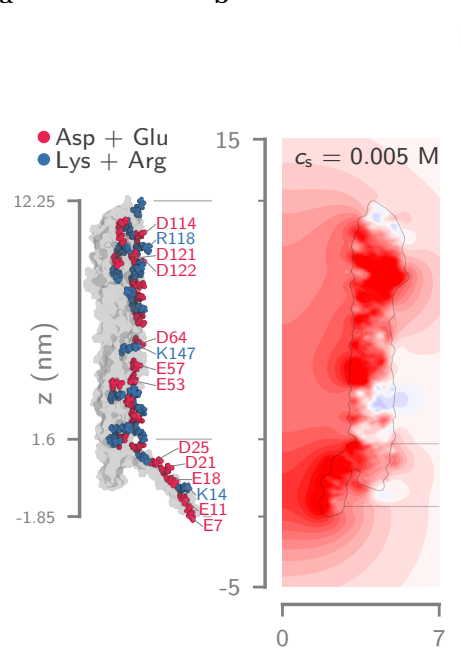

\section{Equilib}
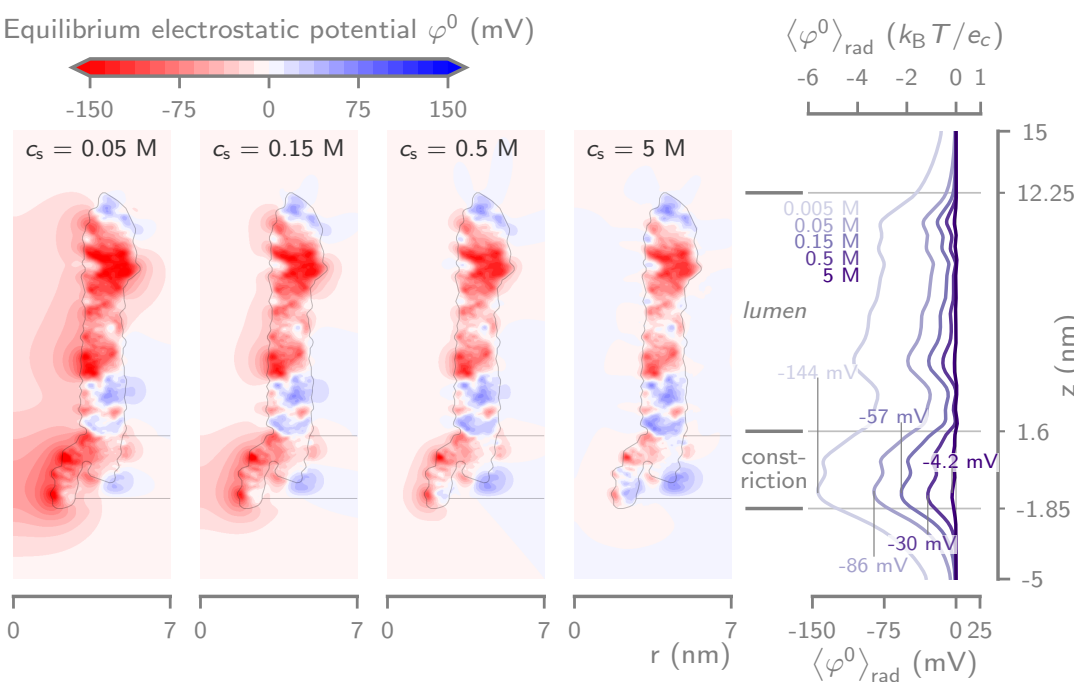

Figure 4. Equilibrium electrostatic potential inside ClyA. a A single subunit of ClyA in which all amino acids with a net charge and whose side chains face the inside of the pore, i.e., those contribute the most to the electrostatic potential, are highlighted. Negatively (Asp+Glu) and positively and positively (Lys+Arg) charged residues are colored in red and blue, respectively. (b) As a result of these fixed charges ClyA exhibits a complex electrostatic potential $(\varphi)$ landscape at equilibrium (i.e., at $\left.V_{\mathrm{b}}=0 \mathrm{mV}\right)$, and whose values inside the pore we have plotted for several key concentrations $\left(c_{\mathrm{s}}=\right.$ $0.005,0.05,0.15,0.5$ and $5 \mathrm{M})$. Note that even at physiological salt concentrations $\left(c_{\mathrm{s}}=0.15 \mathrm{M}\right)$, the negative electrostatic potential extends significantly inside the lumen $(1.6<z<12.25 \mathrm{~nm})$, and even more so inside the trans constriction $(1.85<$ $z<1.6 \mathrm{~nm})$. For the former, localized influential negative 'hotspots' can be found in the middle $(4<z<6 \mathrm{~nm})$ and at the cis entry $(10<z<12 \mathrm{~nm})$. (c) Radial average of the equilibrium electrostatic potential along the length of the pore $\left(\langle\varphi\rangle_{\text {rad }}\right)$ for the same concentrations as in $\left(\mathrm{b}\right.$. Even though the lumen of the pore is almost fully screened for $c_{\mathrm{s}}>0.5 \mathrm{M}$, the constriction still retains some of its negative influence even at $5 \mathrm{M}$.

\section{Electrostatic Potential and Energy}

The electrostatic potential, or rather the change thereof, is one of the primary driving forces within a nanopore. Typically, the potential can be split into an intrinsic, 'equilibrium' component and an external, 'non-equilibrium' contribution. The latter is also known as the bias voltage applied between the trans and the cis reservoirs, and most of it changes across the short distance of the nanopore itself. For a $14 \mathrm{~nm}$-long nanopore such as ClyA, a potential drop of 10 to $100 \mathrm{mV}$ can easily create an electric field of $10^{6}-10^{7} \mathrm{~V} \cdot \mathrm{m}^{-1}$ inside the pore. The intrinsic electrostatic potential results from the charged residues embedded in the interior walls of the protein and can significantly influence the transport of ions and water molecules through the pore. $\frac{461481571120}{1 n}$ the following section we aim to describe the most salient features of this potential and the extent of its influence over the entire investigated concentration range (Fig. 4). Moreover, because electromigration is the primary contributor to the ionic current, a proper understanding of the electrostatic landscape - and the energy 
barriers faced by the ions traversing the pore - should provide a more quantitative explanation for the origin of ClyA's rectification, ion selectivity and asymmetric ion concentrations.

A few important charged residues. The interior walls of the ClyA nanopore (Fig. 4a) are riddled with negatively charged amino amids (i.e., aspargine or glutamine), interspaced by a few positively charged residues (i.e., lysine or arginine). When grouping these charges by proximity, we found three clusters with significantly more negative than positive residues: inside the trans constriction $(-1.85<z<1.6 \mathrm{~nm}$; E7, E11, K14, E18, D21, D25), in the middle of the cis lumen $(4<z<6 \mathrm{~nm}$; E53, E57, D64, K147) and at the top of the pore $(10<z<12 \mathrm{~nm} ; \mathrm{D} 114, \mathrm{R} 118, \mathrm{D} 121, \mathrm{D} 122)$. As we shall see, these clusters leave strong negative fingerprints in the global electrostatic potential.

Distribution of the equilibrium electrostatic potential. The electrostatic potential at equilibrium $\left(\varphi^{0}\right.$, i.e., at $\left.V_{\mathrm{b}}=0 \mathrm{mV}\right)$ reveals the effect of ClyA's fixed charges on the potential inside the pore (Fig. 4b). Due to electric screening by the mobile charge carriers in the electrolyte, however, the extent of their influence strongly depends on the bulk ionic strength. The contour plot cross-sections of $\varphi^{0}$ for $c_{\mathrm{s}}=0.005,0.05,0.15,0.5$ and $5 \mathrm{M}$ (Fig. 4b) and their corresponding radial averages (Fig. 4c) demonstrate this effect aptly. The radial average $\left(\left\langle\varphi^{0}\right\rangle_{\mathrm{rad}}\right)$ represents the mean value along the longitudunal axis of the pore and can be computed using

$$
\langle\varphi\rangle_{\mathrm{rad}}=\frac{1}{\pi R(z)^{2}} \int_{0}^{R(z)} \varphi(r, z) 2 \pi r d r
$$

where $R(z)$ is taken as the pore radius for $-1.85 \leq z \leq 12.25 \mathrm{~nm}, 2 \mathrm{~nm}$ for $z<-1.85 \mathrm{~nm}$ and $4 \mathrm{~nm}$ for $z>12.25 \mathrm{~nm}$. Starting from the cis entry $(z \approx 10 \mathrm{~nm})$, the electrostatic potential is dominated by the acidic residues D114, D121 and D122, resulting in a rapid reduction of $\left\langle\varphi^{0}\right\rangle_{\text {rad }}$ upon entering the pore. Next, $\left\langle\varphi^{0}\right\rangle_{\text {rad }}$ slowly descreases up until the middle of the lumen $(z \approx 5 \mathrm{~nm})$, where the next set of negative residues, namely E53, E57 and D64, lower 
it even further. After a brief increase, $\left\langle\varphi^{0}\right\rangle_{\text {rad }}$ attains its maximum amplitude inside the trans constriction $(z \approx 0 \mathrm{~nm})$ due to the close proximity of the amino acids E7, E11, E18, D21 and D25, and then quickly falls to 0 inside the trans reservoir.

At low ionic strengths $\left(c_{\mathrm{s}}<0.05 \mathrm{M}\right)$, the lack of sufficient ionic screening results in relatively high negative potentials throughout the entire pore. For example, for $c_{\mathrm{s}}=$ 0.005 and $0.05 \mathrm{M}$, the $\left\langle\varphi^{0}\right\rangle_{\text {rad }}$ inside the constriction ramps up to values of $-144 \mathrm{mV}\left(-5.60 \mathrm{k}_{\mathrm{B}} \mathrm{T} / \mathrm{e}\right)$ and $-86 \mathrm{mV}\left(-3.35 \mathrm{k}_{\mathrm{B}} \mathrm{T} / \mathrm{e}\right)$, respectively. These values significantly exceed the single ion thermal voltage $k_{\mathrm{B}} T / e=25.7 \mathrm{mV}$. Hence, on the one hand they prohibit anions such as $\mathrm{Cl}^{-}$from entering the pore, and on the other they attract cations such as $\mathrm{Na}^{+}$and trap them inside the pore. For intermediate concentrations $\left(0.05 \leq c_{\mathrm{s}}<0.5 \mathrm{M}\right)$ the influence of the negative charges becomes increasingly confined to several 'hotspots' near the nanopore walls, most notably at entry of the pore $(10<z<12 \mathrm{~nm})$, in the middle of the lumen $(4<z<6 \mathrm{~nm})$, and in the constriction $(-1.85<z<1.6 \mathrm{~nm})$, in accordance with the charge groups discussed in the previous section. Even though the magnitude of $\left\langle\varphi^{0}\right\rangle_{\mathrm{rad}}$ at $c_{\mathrm{s}}=0.15 \mathrm{M}$ drops below $1 \mathrm{k}_{\mathrm{B}} \mathrm{T} / \mathrm{e}$ inside the lumen $\left(\left\langle\varphi^{0}\right\rangle_{\mathrm{rad}} \approx-14 \mathrm{mV}\right)$, it remains strongly negative inside the constriction $\left(\left\langle\varphi^{0}\right\rangle_{\text {rad }} \approx-47 \mathrm{mV}\right)$. Finally, at high concentrations $\left(c_{\mathrm{s}} \geq 0.5 \mathrm{M}\right)$ the potential is close to $\approx 0 \mathrm{mV}$ over the entire lumen of the pore, with only a small negative potential remaining inside the constriction. A summary of the most salient $\left\langle\varphi^{0}\right\rangle_{\text {rad }}$ values can be found in Tab. S3.

Non-equilibrium electrostatic energy at $+\mathbf{1 5 0}$ and $-\mathbf{1 5 0} \mathbf{m V}$. To link back the observed ionic conductance properties to the electrostatic potential, we computed the radially averaged electrostatic energy for a monovalent ion, $\left\langle U_{\mathrm{E}, i}\right\rangle_{\mathrm{rad}}=z_{i} e\langle\varphi\rangle_{\mathrm{rad}}$, at $V_{\mathrm{b}}=$ $+150-150 \mathrm{mV}$ for the entire range of simulated ionic strengths (Fig. 5a). The resulting energy plot represents the energy landscape — filled with barriers (hills) or traps (valleys) — that a positive or negative ion must traverse in order to contribute positively to (i.e., increase) the ionic current. 
bioRxiv preprint doi: https://doi.org/10.1101/2020.01.08.897819; this version posted January 9, 2020. The copyright holder for this preprint (which was not certified by peer review) is the author/funder, who has granted bioRxiv a license to display the preprint in perpetuity. It is made available under aCC-BY 4.0 International license.
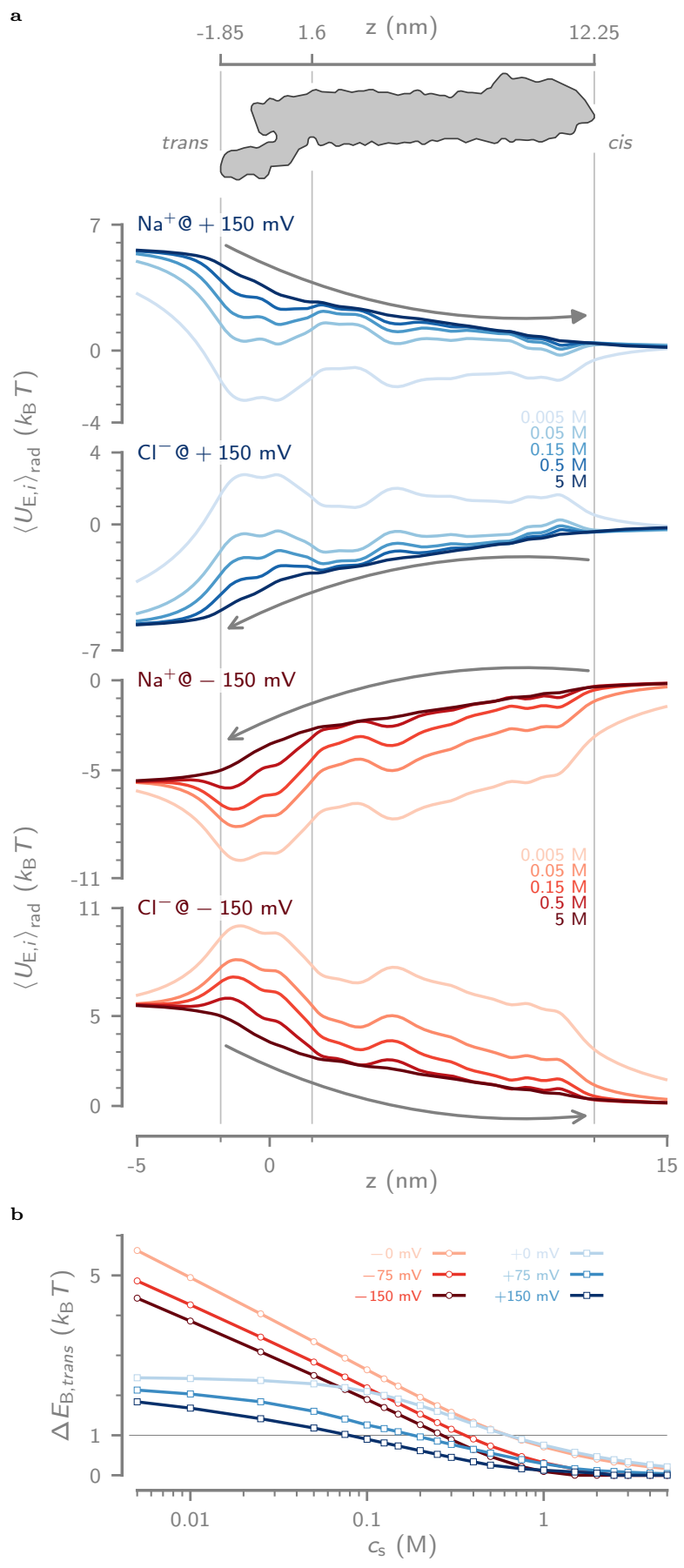

Figure 5. Non-equilibrium electrostatic energy landscape for single ions. a Radially averaged non-equilibrium electrostatic energy landscape for single ions, $\left\langle U_{\mathrm{E}, i}\right\rangle_{\mathrm{rad}}=z_{i} e\langle\varphi\rangle_{\mathrm{rad}}$, as calculated directly from the radial electrostatic potential at $V_{\mathrm{b}}=+150$ and $-150 \mathrm{mV}$ for monovalent cations and anions. The grey arrows indicate the direction in which the ions must travel in order for them to contribute positively to the ionic current. b Height of the electrostatic energy barrier $\left(\Delta E_{\mathrm{B}, i}\right)$ at the trans constriction as a function of the bulk salt concentration. Note that $\Delta E_{\mathrm{B}, i}$ is much higher for negative voltages and rises logarithmically at lower concentrations. The divergence between $+0 \mathrm{mV}$ and $-0 \mathrm{mV}$ for $c_{\mathrm{s}}<0.3 \mathrm{M}$ highlights the difference in barrier height when traversing the pore from cis to trans or vice versa. 
At positive bias voltages, cations traverse the pore from trans to cis (Fig. 5a, first plot). Upon entering the negatively charged constriction, their electrostatic energy drops dramatically, followed by a relatively flat section with a small barrier for entry in the lumen at $z \approx 1.6 \mathrm{~nm}$. At very low ionic strengths $\left(c_{\mathrm{s}}<0.05 \mathrm{M}\right)$, the energy at trans is significantly lower than the energy of the cation in the cis compartment (e.g., $\Delta\left\langle U_{\mathrm{E}, i}\right\rangle_{\mathrm{rad}}>2 \mathrm{k}_{\mathrm{B}} \mathrm{T}$ at $0.005 \mathrm{M}$ ), forcing the ions to accumulate inside the pore. At higher concentrations $\left(c_{\mathrm{s}}>0.05 \mathrm{M}\right)$, the increased screening smooths out the potential drop inside the pore, allowing the cations to migrate unhindered across the entire length of the pore. Anions at $V_{\mathrm{b}}=+150 \mathrm{mV}$ travel from cis to trans (Fig. 5a, second plot) and must overcome energy barriers at both sides of the pore. The former prevents anions such as $\mathrm{Cl}^{-}$from entering the pore. However, because its magnitude is attenuated strongly with increasing salt concentration (from $\approx 1.7 \mathrm{k}_{\mathrm{B}} \mathrm{T}$ at $c_{\mathrm{s}}=0.005 \mathrm{M}$ to $\approx 0.5 \mathrm{k}_{\mathrm{B}} \mathrm{T}$ at $c_{\mathrm{s}}=0.05 \mathrm{M}$ ), it is only relevant at lower ionic strengths $\left(c_{\mathrm{s}}<0.05 \mathrm{M}\right)$. Once inside the lumen, anions can move relatively unencumbered to the trans constriction, where they face the second, more significant energy barrier. This prevents them from fully translocating and causes them to accumulate inside the lumen, explaining the higher $\mathrm{Cl}^{-}$concentrations at positive bias voltages. As with the cations, an increase in the ionic strength significantly reduces these hurdles, resulting in a much smoother landscape for $c_{\mathrm{s}}>0.15 \mathrm{M}$.

At negative voltages, cations move through the pore from cis to trans, with a slow and continuous drop of the electrostatic energy throughout the lumen of the pore up until the constriction (Fig. 5a, third plot). This results in the efficient removal of cations from the pore lumen, and explains the lower $\mathrm{Na}^{+}$concentration observed at positive voltages (Fig. 3a). To fully exit from the pore, however, cations must overcome a large energy barrier, which reduces the nanopore's ability to conduct cations compared to positive potentials and hence contributes to the ion current rectification. The situation for anions at negative bias voltages (i.e., travelling from trans to cis) is very different (Fig. 5a, fourth plot). Their ability to even enter the pore is severely hampered by an energy barrier of a few $\mathrm{k}_{\mathrm{B}} \mathrm{T}$ at the trans 
constriction. Any anions that do cross this barrier, and those still present in the lumen of ClyA, will be rapidly move towards the cis entry and exit from the pore due to a continuous drop of electrostatic energy. This effectively depletes the entire lumen of anions, which can be observed from the much lower $\mathrm{Cl}^{-}$concentrations at negative voltages (see Fig. 3a).

\section{Concentration and voltage dependencies of the energy barrier at the constric-}

tion. Many biological nanopores contain constrictions that play crucial roles in shaping

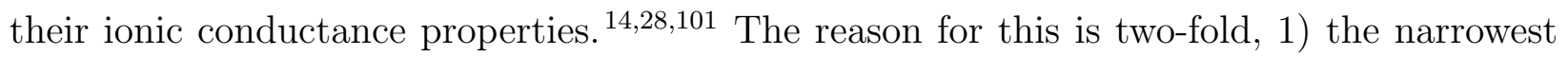
part dominates the overall resistance of the pore and 2) confinement of charged residues results in much larger electrostatic energy barriers. With its highly negatively charged trans constriction, ClyA's affinity for transport of anions is diminished and that for cations is enhanced compared to bulk, even at high ionic strengths (Fig. 2e). ${ }^{96}$ To further elucidate the significance of the trans electrostatic barrier $\left(\Delta E_{\mathrm{B}, i}\right)$, we quantified its height at positive and negative voltages as a function of the salt concentration (Fig. 5b).

Due to the tilting of the nergy landscape, the application of a bias voltage lowers the magnitude of the energy barrier for all conditions. Likewike, increasing the bulk salt concentration results in a continuous decrease of $\Delta E_{\mathrm{B}, i}$. For $c_{\mathrm{s}}>0.5 \mathrm{M}, \Delta E_{\mathrm{B}, i}$ falls below $1 \mathrm{k}_{\mathrm{B}} \mathrm{T}$ regardless of the bias voltage, reducing its effect on the ion transport through the pore. The barrier heights for ions under the influence of a positive bias voltage (i.e., $\mathrm{Na}^{+}$moving from trans to cis and $\mathrm{Cl}^{-}$moving from cis to trans), experience a $\Delta E_{\mathrm{B}, i}$ roughly half that of those under a negative voltage. For example, upon increasing the salt concentration from 0.005 to $0.15 \mathrm{M}, \Delta E_{\mathrm{B}, i}$ dropped from 1.8 to $0.73 \mathrm{k}_{\mathrm{B}} \mathrm{T}$ at $V_{\mathrm{b}}=+150 \mathrm{mV}$ and from 4.4 to $1.5 \mathrm{k}_{\mathrm{B}} \mathrm{T}$ at $V_{\mathrm{b}}=-150 \mathrm{mV}$. This explains the differences in ion selectivity observed between positive and negative bias voltages (Fig. 2e). At $c_{\mathrm{s}}=0.15 \mathrm{M}$, for example, the ion selectivity $t_{\mathrm{Na}^{+}}$ increases from 0.79 at $V_{\mathrm{b}}=+150 \mathrm{mV}$ to 0.88 at $V_{\mathrm{b}}=-150 \mathrm{mV}$. 
bioRxiv preprint doi: https://doi.org/10.1101/2020.01.08.897819; this version posted January 9, 2020. The copyright holder for this preprint (which was not certified by peer review) is the author/funder, who has granted bioRxiv a license to display the preprint in perpetuity. It is made available under aCC-BY 4.0 International license.

a
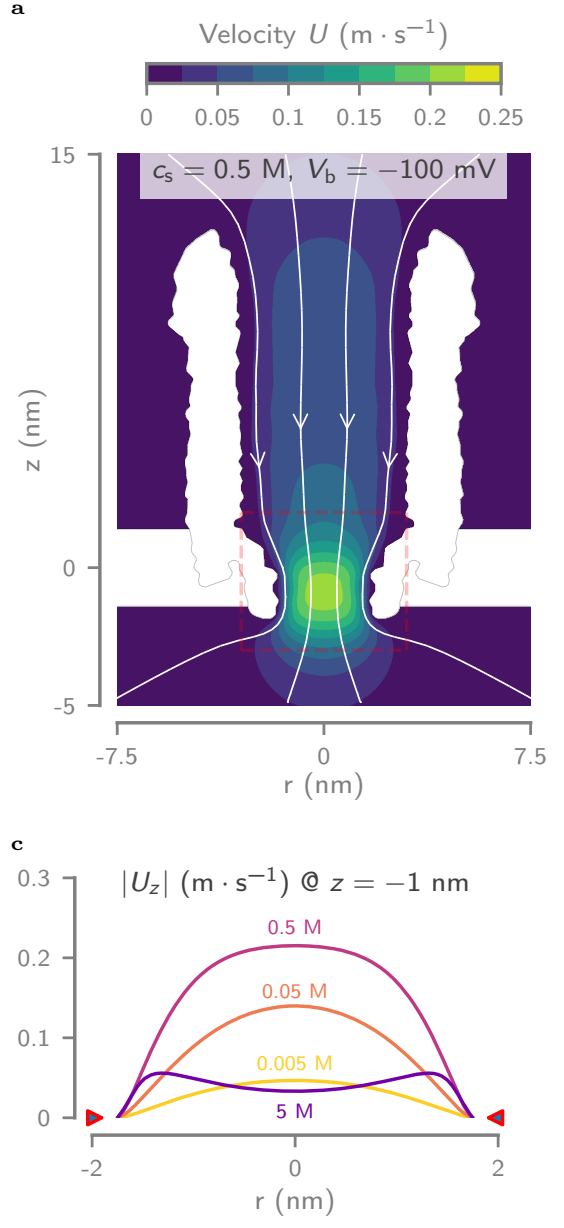

b

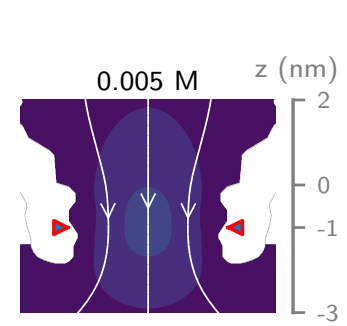

$0.05 \mathrm{M}$

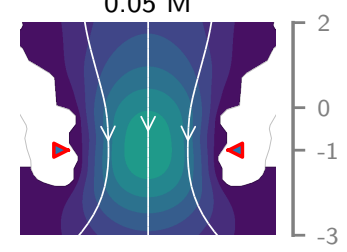

$0.5 \mathrm{M}$

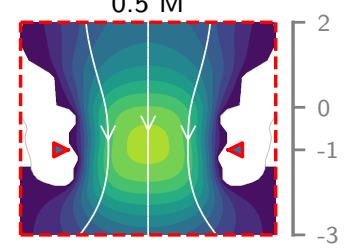

$5 \mathrm{M}$

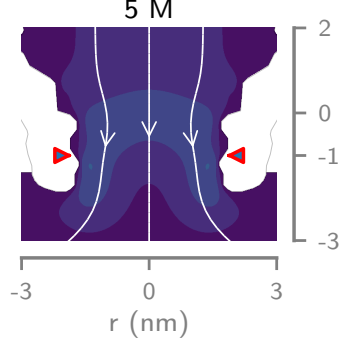

d

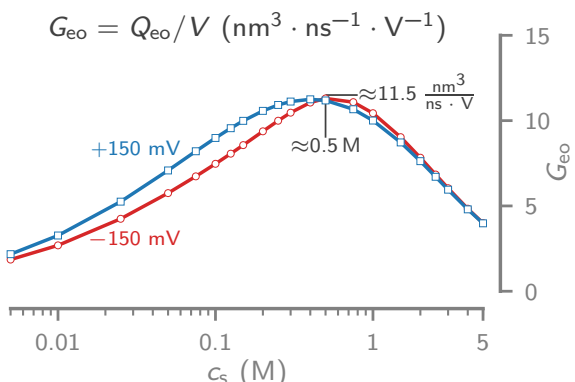

e

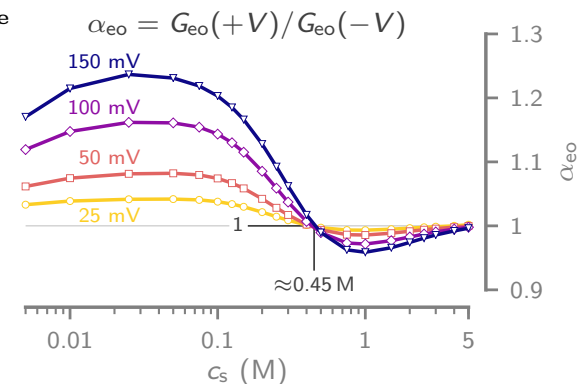

Figure 6. Concentration and voltage dependency of the electro-osmotic flow inside ClyA. a Contour plot of the electro-osmotic flow (EOF) velocity $\boldsymbol{u}$ at $0.5 \mathrm{M}$ and $-100 \mathrm{mV}$ bias voltage. The arrows on the streamlines indicate the direction of the flow. As observed experimentally $[96$ and expected from a negatively charged conical nanopore, the EOF follows the direction of the cation, i.e., from cis to trans under negative bias voltages and vice versa for positive ones. (b) Contour plots of the EOF field in the trans constriction for various salt concentrations at $V_{\mathrm{b}}=-100 \mathrm{mV}$ and (c) cross-sections of the absolute value of the water velocity $\left|U_{z}\right|$ at $z=-1 \mathrm{~nm}$. Notice that at high salt concentrations $\left(c_{\mathrm{s}}>1 \mathrm{M}\right)$, the velocity profile exhibits two 'lobes' close to the nanopore walls and hence deviates from the parabolic shape observed at lower ionic strengths. (d) Concentration dependence of the electro-osmotic conductance $G_{\mathrm{eo}}=Q_{\mathrm{eo}} / V_{\mathrm{b}}$, with $Q_{\mathrm{eo}}$ the total flow rate through the pore (Eq. 21). In the low concentration regime, $G_{\text {eo }}$ increases rapidly between 0.005 and $0.5 \mathrm{M}$ after which it decreases logarithmically for higher concentrations. (e) The rectification of the electro-osmotic conductance $\left(\alpha_{\mathrm{eo}}(V)=G_{\mathrm{eo}}(+V) / G_{\mathrm{eo}}(-V)\right)$ plotted against the bulk salt concentration. The $\alpha_{\mathrm{eo}}$ increases with bias voltage and exhibits an inversion point at $c_{\mathrm{s}} \approx 0.45 \mathrm{M}$. 


\section{Transport of Water Through ClyA}

The charged nature of many nanopores gives rise to a net flux of water through the pore, called the electro-osmotic flow (EOF). 37821121 The EOF not only contributes significantly to the ionic current, but the magnitude of the viscous drag force it exerts is often of the same order as the Coulombic electrophoretic force (EPF). Hence, it strongly influences the capture and translocation of biomolecules including nucleic acids, $\stackrel{36 / 47122]}{\text { peptides, }}, \underline{2811231124}$ and proteins. $.2512730196 \mid 99125$ Because the drag exerted by the EOF depends primarily on the size and shape of the biomolecule of interest and not on its charge, $\frac{125}{1 t}$ can be harnassed to capture molecules even against the electric field. ${ }^{25}$ The EOF is a consequence of interaction between the fixed charges on the nanopore walls and mobile charges in the electrolyte. It can be described by two closely related mechanisms: 1) the excess transport of the hydration shell water molecules in one direction due to the pore's ion selectivity, and 2) the viscous drag exerted by the unidirectional movement of the electrical double layer inside the pore. The first mechanism likely dominates in pores with a diameter close to that of the hydrated ions $(\leq 1 \mathrm{~nm})$ such as $\alpha \mathrm{HL}$ or FraC, $\stackrel{281124}{ }$ while the second is expected to be stronger for larger pores $(>1 \mathrm{~nm})$, such as ClyA 251125 or most solid-state nanopores. $\frac{\sqrt{3739}}{2 n}$ our simulation, the EOF follows the coupling of the Navier-Stokes and the Poisson-Nernst-Planck equations through a volume force term (Eq. 13), which dictates that the electric field exerts a net force on the fluid if it contains a net charge density - as is the case for the electrical double layer lining the walls of ClyA (Fig. 3d). In the following section we aim to quantitatively and a qualitatively describe the properties of the water velocity inside ClyA and how it is influenced by the bulk ionic strength and the applied bias voltage (Fig. 6).

Direction, magnitude and distribution of the water velocity. As expected, given ClyA's negatively charged interior surface and the resulting positively charged electrical double layer, the direction of the net water flow inside ClyA follows the electric field, i.e., from the cis to the trans at negative bias voltages (Fig. 6a). This corresponds to the observations 
and analysis of single-molecule protein capture ${ }^{\sqrt{96}}$ and trapping $\sqrt{97 / 98 \mid 125}$ experiments using the ClyA-AS nanopore. Along the longitudinal axis $(z)$ of the pore, the water velocity is governed by the conservation of mass, meaning it is lowest in the wide cis lumen and highest in the narrow trans constriction (Fig. 6a). For example, at $V_{\mathrm{b}}=-100 \mathrm{mV}$ and $c_{\mathrm{s}}=0.5 \mathrm{M}$ the velocity at the center of the pore is $\approx 0.07 \mathrm{~m} \cdot \mathrm{s}^{-1}$ in the lumen and $\approx 0.21 \mathrm{~m} \cdot \mathrm{s}^{-1}$ in the constriction.

Along the radial axis $(r), \boldsymbol{u}$ has a parabolic profile with the highest value at the center of the pore and the lowest at the wall due to the no-slip boundary condition (Figs. 6b and 6c). Such a parabolic profile contrasts the expected 'plug flow' for an EOF, but follows logically from the overlap of the electrical double layer inside the pore and the resulting uniform volume force - analogous to a gravity- or pressure-driven Stokes flow. At concentrations higher than 0.5 M, however, the increasing degree of confinement of the double layer-and its charge - to the nanopore walls (see Fig. $3 \mathrm{~d}$, $\left\langle Q_{\text {ion }}\right\rangle_{\mathrm{s}}$ ) results in a flattening of the central maximum and hence a plug flow profile. Interestingly, at very high salt concentrations $\left(c_{\mathrm{s}} \geq 1 \mathrm{M}\right)$ the velocity profile in the constriction exhibits a dimple at the center of the pore. This is the result of a self-induced pressure gradient caused by the expansion of the EOF as it exits the pore. $\frac{126}{}$

\section{Influence of bulk ionic strength and bias voltage on the electro-osmotic conduc-}

tance. In analogy to the ionic conductance, the amount of water transported by ClyA can be expressed by the electro-osmotic conductance $G_{\mathrm{eo}}=Q_{\mathrm{eo}} / V_{\mathrm{b}}$ (Fig. $6 \mathrm{~d}$ ). Here, $Q_{\mathrm{eo}}$ is the net volumetric flow rate of water through the pore and computed by integrating the water velocity across the reservoir boundary (Eq. 21). The strength of the EOF depends strongly and non-monotonically on the bulk ionic strength: $G_{\mathrm{eo}}$ rapidly increases with ionic strength until a peak value is reached at $c_{\mathrm{s}} \approx 0.5 \mathrm{M}$, followed by a gradual logarithmic decline (Fig. 6d). For example, at $V_{\mathrm{b}}=-150 \mathrm{mV}, G_{\mathrm{eo}}$ changes from $1.85,11.3$ to $4.00 \mathrm{~nm}^{3} \cdot \mathrm{ns}^{-1} \cdot \mathrm{V}^{-1}$ at for $c_{\mathrm{s}}=0.005,0.5$ and $5 \mathrm{M}$, respectively. 
The sensitivity of the EOF to the magnitude and sign of the bias voltage is given by the electro-osmotic conductance rectification $\alpha_{\mathrm{eo}}(V)=G_{\mathrm{eo}}(+V) / G_{\mathrm{eo}}(-V)$ (Fig. 6e). For all voltages magnitudes, $\alpha_{\mathrm{eo}}$ shows a maximum at $c_{\mathrm{s}} \approx 0.045 \mathrm{M}$, after which it falls rapidly to reach unity $\left(\alpha_{\mathrm{eo}}=1\right)$ at approximately $c_{\mathrm{s}} \approx 0.45 \mathrm{M}$. A minimum is then reached at $\approx 1 \mathrm{M}$, followed by a gradual approach towards unity at $c_{\mathrm{s}}=5 \mathrm{M}$.

\section{Conclusions}

We have developed an extended version of the Poison-Nernst-Planck-Navier-Stokes (ePNPNS) equations and used it to model the transport of ions and water through the biological nanopore ClyA. Our ePNP-NS equations combine many of the improvements to the PNP-NS equations available in literature, in addition to several new corrections. These include the finite size of the ions, self-consistent concentration- and positional-dependent parametrization of the ionic transport coefficients (diffusion coefficient and mobility) and of the electrolyte properties (density, viscosity and relative permittivity).

We have verified our approach by matching experimental results to a very high degree of accuracy and the model parameters where gauged on other experiments, ultimately leaving no degrees of freedom. This shows that a continuum approach to modeling biological nanopores is not only feasible but to a very high degree predictive. We are confident that such a model as presented in this paper, constitutes a highly practical tool that can help with 1) elucidating the link between ionic current observed during a nanopore experiment and the actual physical phenomenon, 2) describing the electrophoretic and electro-osmotic properties of any biological nanopore and 3) guiding the design of new variants of existing nanopores.

In the analysis of the operation of ClyA with our model we found that the ionic currents depend strongly on the details of the electric field structure within the pore which define potential barriers for the ion species. These barriers in turn are influenced by both the 
bulk ionic strength and the applied bias voltage. Nevertheless, despite its simplified 2Daxisymmetric nature, our model is able to obtain meaningful and predictive results in terms of the ionic current, the ion concentrations, the electrostatic potential and electro-osmotic flow velocity. Some inaccuracies still arise at low salt concentrations, but these can likely be resolved with an improved mobility model.

Although not explicitly investigated in the current work, there are no obvious reasons why the model framework is not directly applicable to other biological nanopores, solid-state nanopores and other nanoscale ionic transport problems. These systems can be treated analogously and their properties could be mapped out systematically using our ePNP-NS model, greatly enhancing the understanding and interpretation of experimental results.

\section{Materials and Methods}

ClyA-AS homology model. A full atom model of ClyA-AS ${ }^{\sqrt{96}}$ was built and optimized (MODELLER v9.18 $8^{127}$ ) by introduction of the following point mutations in each of the 12 chains of the wild-type ClyA crystal structure (PDBID: 2WCD ${ }^{95}$ ): K8Q, N15S, Q38K, A57G, T67V, C87A, A90V, A95S, L99Q, E103G, K118R, L119I, I124V, T125K, V136T, F166Y, K172R, V185I, K212N, K214R, S217T, T224S, N227A, T244A, E276G, C285S, K290Q. Next, the conformation of all mutated side chains was optimized with an double annealing protocol (heating: 150, 250, 400, 700 and $1000 \mathrm{~K}$, cooling: 1000, 800, 600, 500, 400 and $300 \mathrm{~K}$ ) where at each temperature the energy was minimized for 200 iterations with a conjugate gradients algorithm (4 fs timestep). $\frac{128}{1}$ The first anneal was performed solely on the mutated residues themselves, and the second run also took the non-bonded interactions with the neighboring atoms into account. The refined nanopore structure was then embedded in the center of an $18 \mathrm{~nm} \times 18 \mathrm{~nm}$ equilibrated DPhPC lipid bilayer patch by manual removal of all overlapping lipids, resulting in 463 lipid molecules. The bilayer was created with the CHARMM-GUI 129 membrane builder ${ }^{130}$ and equilibrated with $\mathrm{NAMD}^{131}$, as described in detail in ref. [ 132]. 
The system was then solvated in a box of $18 \mathrm{~nm} \times 18 \mathrm{~nm} \times 32 \mathrm{~nm}$ by addition of 214640 TIP 3 water molecules (VMD solvate plugin), and the global charge was neutralized by replacing 1276 random water molecules with $674 \mathrm{Na}^{+}$and $602 \mathrm{Cl}^{-}$ions (VMD autoionize plugin). 102

Molecular dynamics simulations. Using molecular dynamics (MD) with NAMD 2.12 (2 fs timestep, CHARMM36 forcefield ${ }^{133}$ ), the final system was minimized for 5 ps, heated from 0 to $298.15 \mathrm{~K}$ in $4 \mathrm{ps}$ and equilibrated for $4 \mathrm{~ns}$ as NpT ensemble. ${ }^{46}$ Finally a 30 ns production run was performed using a NVT ensemble at $298.15 \mathrm{~K}$ and the atomic coordinates saved every 5 ps. Note that structural deterioration was prevented by harmonically restraining the protein's $\mathrm{C}_{\alpha}$ atoms to their original positions (spring constant of $695 \mathrm{pN} \cdot \mathrm{nm}^{-1}$ ) during all MD runs. $\underline{48}$

Axially symmetric geometry. The 2D-axisymmetric geometry of the ClyA-AS nanopore (Fig. 1c) was derived directly from its full atom model by radially averaging the molecular density. Briefly, 50 sets of atomic coordinates were extracted from the final $5 \mathrm{~ns}$ of the coordinates of the $30 \mathrm{~ns}$ MD production run (i.e., every $100 \mathrm{fs}$ ) and aligned by minimizing the RMSD between their backbone atoms (VMD RMSD tool). Next, we computed and averaged the 3D-dimensional molecular density maps of all 50 structures on a $0.5 \AA$ resolution grid using the Gaussian function 104

$$
\rho_{\mathrm{mol}}=1-\prod_{i}\left[1-\exp \left(-\frac{-d_{i}^{2}}{\left(\sigma R_{i}\right)^{2}}\right)\right]
$$

where for each atom $i, R_{i}$ is its Van der Waals radius, $d_{i}=\sqrt{\left(x-x_{i}\right)^{2}+\left(y-y_{i}\right)^{2}+\left(z-z_{i}\right)^{2}}$ is the distance of grid coordinates $(x, y, z)$ from the atom center $\left(x_{i}, y_{i}, z_{i}\right)$ and $\sigma=0.93$ is a width factor. The resulting 3D density map was then radially averaged along the z-axis, relative to the center of the pore to obtain a 2D-axisymmetric density map. The contourline at $25 \%$ density was used as the nanopore simulation geometry, after manual removal of overlapping and superfluous vertices to improve the quality of the final computational mesh. 
Axially symmetric charge density. The 2D-axially symmetric charge distribution (Fig. 1d was also derived directly from the 50 sets of aligned nanopore coordinates) that were used for the geometry. Inspired by how charges are represented in the particle mesh Ewald (PME) method, $\stackrel{46}{a}$ we computed the fixed charge distribution of the nanopore $\rho_{\text {pore }}^{f}(r, z)$ by assuming that an atom $i$ of partial charge $\delta_{i}$, at the location $\left(x_{i}, y_{i}, z_{i}\right)$ in the full 3D atomistic pore model, contributes an amount $\delta_{i} / 2 \pi r_{i}$ to the partial charge at a point $\left(r_{i}, z_{i}\right)$ with $r_{i}=\sqrt{x_{i}^{2}+y_{i}^{2}}$ in the averaged 2D-axisymmetric model. This effectively spreads the charge over all angles to achieve axial symmetry. We assumed a Gaussian distribution of the space charge density of each atom $i$ around its respective 2D-axisymmetric coordinates $\left(r_{i}, z_{i}\right)$ is

$$
\rho_{\text {pore }}^{f}(r, z)=\sum_{i} \frac{e \delta_{i}}{\pi\left(\sigma R_{i}\right)^{2}} \exp \left(-\frac{\left(r-r_{i}\right)^{2}+\left(z-z_{i}\right)^{2}}{\left(\sigma R_{i}\right)^{2}}\right)
$$

where $R_{i}$ is the atom radius, $\sigma=0.5$ is the sharpness factor and $e$ is the elementary charge. To embed $\rho_{\text {pore }}^{f}$ with sufficient detail, yet efficiently, into a numeric solver, the spatial coordinates were discretized with a grid spacing of $0.005 \mathrm{~nm}$ in the domain of $\rho_{\text {pore }}^{f}$ and precomputed values were used during the solver runtime. All partial charges (at pH 7.5) and radii were taken from the CHARMM36 forcefield ${ }^{133}$ and assigned using PROPKA 134 and PBD2PQR. 135

Computing electrophoretic mobilities. To obtain the concentration-dependent ionic mobility $\mu_{i}^{c}$ from fitted functions, it must first be derived from the salt's molar conductivity $\Lambda$ and the ion's transport number $t_{i}$ before it can be fitted ${ }^{86}$

$$
\mu_{i}(c)=\frac{\lambda_{i}(c)}{z_{i} \mathcal{F}} \quad \text { with } \quad \lambda_{i}(c)=\Lambda(c) t_{i}(c)
$$

where $\lambda_{i}(c)$ is the specific molar conductivity of ion $i$. 
Computing the simulated ionic current and electro-osmotic flow rate. The simulated ionic current $I_{\text {sim }}$ at steady-state was computed by

$$
I_{\text {sim }}=\mathcal{F} \int_{S}\left(\sum_{i} z_{i} \hat{\boldsymbol{n}} \cdot \boldsymbol{J}_{i}\right) d S,
$$

with $z_{i}$ the charge number and $\boldsymbol{J}_{i}$ the total flux of each ion $i$ across cis reservoir boundary $S, \mathcal{F}$ the Faraday constant $\left(96485 \mathrm{C} \cdot \mathrm{mol}^{-1}\right)$ and $\hat{\boldsymbol{n}}$ the unit vector normal to $S$. Similarly, the volumetric flow rate, i.e., the volume of water passing through the pore per unit time, is given by

$$
Q_{\mathrm{eo}}=\int_{S}\left(\sum_{i} \hat{\boldsymbol{n}} \cdot \boldsymbol{u}\right) d S
$$

ClyA expression and purification. ClyA-AS monomers were expressed, purified and oligomerized using methods described in detail elsewhere. ${ }^{25196}$ Briefly, E. cloni EXPRESS BL21 (DE3) cells (Lucigen Corporation, Middleton, USA) were transformed with a pT7 plasmid containing the ClyA-AS gene, followed by overexpression after induction with $1 \mathrm{mM}$ isopropyl $\beta$-D-1-thiogalactopyranoside (Sigma-Aldrich, Zwijndrecht, The Netherlands). The ClyA monomers were purified using $\mathrm{Ni}^{+}$-NTA affinity chromatography and oligomerized by incubation in $0.2 \%$ D-maltoside n-dodecyl- $\beta$-D-maltopyranoside (Sigma-Aldrich, Zwijndrecht, The Netherlands) for 30 minutes at $37^{\circ} \mathrm{C}$. Pure ClyA-AS type-I (12-mer) nanopores were obtained using native PAGE on a 4-15\% gradient gel (Bio-Rad, Veenendaal, The Netherlands) and subsequent excision of the correct oligomer band.

Recording of single-channel current-voltage curves. Experimental current-voltage curves where measured using single-channel electrophysiology, as detailed elsewhere. $\frac{25|35| 96}{20}$ Briefly, a black lipid bilayer was inside a $\approx 100 \mu \mathrm{m}$ diameter aperture in a thin teflon film separating two buffered electrolyte compartments by painting with 1,2-diphytanoyl-snglycero-3phosphocholine (DPhPC, Avanti Polar Lipids, Alabaster, USA). Minute amounts $(\approx)$ of the 
purified ClyA-AS type I oligimer were then added to the grounded cis reservoir and allowed to insert into the lipid bilayer. Single-channel current-voltage curves were recorded using a custom pulse protocol of the Clampex 10.4 software package connected to AxoPatch 200B patch-clamp amplifier via a Digidata 1440A digitizer (all from Molecular Devices, San Jose, USA). Data was acquired at $10 \mathrm{kHz}$ and filtered using a $2-\mathrm{kHz}$ low bandpass filter. Measurements at different ionic strengths were performed at $\approx 25^{\circ} \mathrm{C}$ in aqueous $\mathrm{NaCl}$ solutions, buffered at pH 7.5 using 10 mM MOPS (Sigma-Aldrich, Zwijndrecht,The Netherlands).

\section{Acknowledgement}

K.W., J.H. and P.V.D. acknowledge the financial support by the FWO (grant number 3E130054). G.M. has received funding from the European Research Council (ERC) under the European Union's Horizon 2020 research and innovation programme (Grant agreement No. 726151). The authors thank Niels Verellen and Chang Chen for their valuable feedback during discussions.

\section{Supporting Information Available}

The supplementary info contains the Extended materials and methods, with details on the fitting of the electrolyte properties and the calculation of the pore averaged values, and the weak forms of the ePNP-NS equations. It also contains additional results, including a figures about ionic current rectification and the electro-osmotic pressure distribution inside the pore, and a table detailing the peak values of the radial electrostatic potential inside ClyA.

\section{References}

1. Sparreboom, W.; van den Berg, A.; Eijkel, J. C. T. Transport in nanofluidic systems: a review of theory and applications. New Journal of Physics 2010, 12, 015004. 
2. Bocquet, L.; Charlaix, E. Nanofluidics, from bulk to interfaces. Chemical Society Reviews 2010, 39, 1073-95.

3. Maffeo, C.; Bhattacharya, S.; Yoo, J.; Wells, D.; Aksimentiev, A. Modeling and simulation of ion channels. Chemical Reviews 2012, 112, 6250-84.

4. Thomas, M.; Corry, B.; Hilder, T. A. What Have We Learnt About the Mechanisms of Rapid Water Transport, Ion Rejection and Selectivity in Nanopores from Molecular Simulation? Small 2014, 10, 1453-1465.

5. Wang, J.; Zhang, M.; Zhai, J.; Jiang, L. Theoretical simulation of the ion current rectification (ICR) in nanopores based on the Poisson-Nernst-Planck (PNP) model. Physical Chemistry Chemical Physics : PCCP 2014, 16, 23-32.

6. Kim, H. S.; Kim, Y.-H. Recent progress in atomistic simulation of electrical current DNA sequencing. Biosensors \& bioelectronics 2015, 69, 186-98.

7. Bayley, H.; Cremer, P. S. Stochastic sensors inspired by biology. Nature 2001, 413, 226-30.

8. Dekker, C. Solid-state nanopores. Nature Nanotechnology 2007, 2, 209-15.

9. Venkatesan, B. M.; Bashir, R. Nanopore sensors for nucleic acid analysis. Nature Nanotechnology 2011, 6, 615-24.

10. Zhang, A.; Lieber, C. M. Nano-Bioelectronics. Chemical Reviews 2016, 116, 215-57.

11. Deamer, D.; Akeson, M.; Branton, D. Three decades of nanopore sequencing. Nature Biotechnology 2016, 34, 518-24.

12. Kasianowicz, J. J.; Brandin, E.; Branton, D.; Deamer, D. W. Characterization of individual polynucleotide molecules using a membrane channel. Proceedings of the National Academy of Sciences of the United States of America 1996, 93, 13770-3.

13. Meller, A.; Nivon, L.; Brandin, E.; Golovchenko, J.; Branton, D. Rapid nanopore discrimination between single polynucleotide molecules. Proceedings of the National Academy of Sciences of the United States of America 2000, 97, 1079-84. 
14. Maglia, G.; Restrepo, M. R.; Mikhailova, E.; Bayley, H. Enhanced translocation of single DNA molecules through $\alpha$-hemolysin nanopores by manipulation of internal charge. Proceedings of the National Academy of Sciences of the United States of America 2008, 105, 19720-5.

15. Butler, T. Z.; Pavlenok, M.; Derrington, I. M.; Niederweis, M.; Gundlach, J. H. Singlemolecule DNA detection with an engineered MspA protein nanopore. Proceedings of the National Academy of Sciences of the United States of America 2008, 105, 20647-52.

16. Stoddart, D.; Heron, A. J.; Mikhailova, E.; Maglia, G.; Bayley, H. Single-nucleotide discrimination in immobilized DNA oligonucleotides with a biological nanopore. Proceedings of the National Academy of Sciences of the United States of America 2009, 106, 7702-7.

17. Franceschini, L.; Soskine, M.; Biesemans, A.; Maglia, G. A nanopore machine promotes the vectorial transport of DNA across membranes. Nature Communications 2013, 4, 2415.

18. Jain, M.; Koren, S.; Miga, K. H.; Quick, J.; Rand, A. C.; Sasani, T. A.; Tyson, J. R.; Beggs, A. D.; Dilthey, A. T.; Fiddes, I. T.; Malla, S.; Marriott, H.; Nieto, T.; O, B.; dy, J.; Olsen, H. E.; Pedersen, B. S.; Rhie, A.; Richardson, H.; Quinlan, A. R.; Snutch, T. P.; Tee, L.; Paten, B.; Phillippy, A. M.; Simpson, J. T.; Loman, N. J.; Loose, M. Nanopore sequencing and assembly of a human genome with ultra-long reads. Nature Biotechnology 2018, 36, 338-345.

19. Restrepo-Pérez, L.; Joo, C.; Dekker, C. Paving the way to single-molecule protein sequencing. Nature Nanotechnology 2018, 13, 786-796.

20. Talaga, D. S.; Li, J. Single-molecule protein unfolding in solid state nanopores. Journal of the American Chemical Society 2009, 131, 9287-97.

21. Rodriguez-Larrea, D.; Bayley, H. Multistep protein unfolding during nanopore translocation. Nature Nanotechnology 2013, 8, 288-95.

22. Nivala, J.; Marks, D. B.; Akeson, M. Unfoldase-mediated protein translocation through an ג-hemolysin nanopore. Nature Biotechnology 2013, 31, 247-50. 
23. Kennedy, E.; Dong, Z.; Tennant, C.; Timp, G. Reading the primary structure of a protein with $0.07 \mathrm{~nm}$ resolution using a subnanometre-diameter pore. Nature Nanotechnology 2016, 11, 968-976.

24. Chen, C.; Xie, Q.; Yang, D.; Xiao, H.; Fu, Y.; Tan, Y.; Yao, S. Recent advances in electrochemical glucose biosensors: a review. RSC Advances 2013, 3, 4473.

25. Soskine, M.; Biesemans, A.; Moeyaert, B.; Cheley, S.; Bayley, H.; Maglia, G. An engineered ClyA nanopore detects folded target proteins by selective external association and pore entry. Nano Letters 2012, 12, 4895-900.

26. Niedzwiecki, D. J.; Iyer, R.; Borer, P. N.; Movileanu, L. Sampling a biomarker of the human immunodeficiency virus across a synthetic nanopore. ACS Nano 2013, 7, 3341-50.

27. Van Meervelt, V.; Soskine, M.; Maglia, G. Detection of two isomeric binding configurations in a protein-aptamer complex with a biological nanopore. ACS Nano 2014, 8, 12826-35.

28. Huang, G.; Willems, K.; Soskine, M.; Wloka, C.; Maglia, G. Electro-osmotic capture and ionic discrimination of peptide and protein biomarkers with FraC nanopores. Nature Communications 2017, 8, 935 .

29. Liu, L.; Li, T.; Zhang, S.; Song, P.; Guo, B.; Zhao, Y.; Wu, H.-C. Simultaneous Quantification of Multiple Cancer Biomarkers in Blood Samples through DNA-Assisted Nanopore Sensing. Angewandte Chemie 2018, 130, 12058-12063.

30. Galenkamp, N. S.; Soskine, M.; Hermans, J.; Wloka, C.; Maglia, G. Direct electrical quantification of glucose and asparagine from bodily fluids using nanopores. Nature Communications 2018, 9, 4085.

31. Willems, K.; Van Meervelt, V.; Wloka, C.; Maglia, G. Single-molecule nanopore enzymology. Philosophical Transactions of the Royal Society of London. Series B, Biological Sciences 2017, 372. 
32. Lieberman, K. R.; Cherf, G. M.; Doody, M. J.; Olasagasti, F.; Kolodji, Y.; Akeson, M. Processive replication of single DNA molecules in a nanopore catalyzed by phi29 DNA polymerase. Journal of the American Chemical Society 2010, 132, 17961-72.

33. Ho, C.-W.; Van Meervelt, V.; Tsai, K.-C.; De Temmerman, P.-J.; Mast, J.; Maglia, G. Engineering a nanopore with co-chaperonin function. Science Advances 2015, 1, e1500905.

34. Laszlo, A. H.; Derrrington, I. M.; Gundlach, J. H. Subangstrom Measurements of Enzyme Function Using a Biological Nanopore, SPRNT. Methods in Enzymology 2017, 582, 387-414.

35. Maglia, G.; Heron, A. J.; Stoddart, D.; Japrung, D.; Bayley, H. Analysis of Single Nucleic Acid Molecules with Protein Nanopores. 2010, 475, 591-623.

36. Wong, C. T. A.; Muthukumar, M. Polymer capture by electro-osmotic flow of oppositely charged nanopores. The Journal of Chemical Physics 2007, 126, 164903.

37. Mao, M.; Sherwood, J. D.; Ghosal, S. Electro-osmotic flow through a nanopore. Journal of Fluid Mechanics 2014, 749, 167-183.

38. Haywood, D. G.; Harms, Z. D.; Jacobson, S. C. Electroosmotic flow in nanofluidic channels. Analytical Chemistry 2014, 86, 11174-80.

39. Laohakunakorn, N.; Keyser, U. F. Electroosmotic flow rectification in conical nanopores. Nanotechnology 2015, 26, 275202.

40. Grosberg, A. Y.; Rabin, Y. DNA capture into a nanopore: interplay of diffusion and electrohydrodynamics. The Journal of Chemical Physics 2010, 133, 165102.

41. Muthukumar, M. Theory of capture rate in polymer translocation. The Journal of Chemical Physics 2010, 132, 195101.

42. Muthukumar, M. Communication: Charge, diffusion, and mobility of proteins through nanopores. The Journal of Chemical Physics 2014, 141, 081104. 
43. Yusko, E. C.; Bruhn, B. R.; Eggenberger, O. M.; Houghtaling, J.; Rollings, R. C.; Walsh, N. C.; Nandivada, S.; Pindrus, M.; Hall, A. R.; Sept, D.; Li, J.; Kalonia, D. S.; Mayer, M. Realtime shape approximation and fingerprinting of single proteins using a nanopore. Nature Nanotechnology 2017, 12, 360-367.

44. Lynden-Bell, R. M.; Rasaiah, J. C. Mobility and solvation of ions in channels. The Journal of Chemical Physics 1996, 105, 9266-9280.

45. Allen, T. W.; Kuyucak, S.; Chung, S.-H. The effect of hydrophobic and hydrophilic channel walls on the structure and diffusion of water and ions. Journal of Chemical Physics 1999, $111,7985-7999$.

46. Aksimentiev, A.; Schulten, K. Imaging alpha-hemolysin with molecular dynamics: ionic conductance, osmotic permeability, and the electrostatic potential map. Biophysical Journal 2005, 88, 3745-61.

47. Luan, B.; Aksimentiev, A. Electro-osmotic screening of the DNA charge in a nanopore. Physical Review E 2008, 78, 021912.

48. Bhattacharya, S.; Muzard, L.; Payet, L.; Mathé, J.; Bockelmann, U.; Aksimentiev, A.; Viasnoff, V. Rectification of the current in alpha-hemolysin pore depends on the cation type: the alkali series probed by MD simulations and experiments. The Journal of Physical Chemistry C 2011, 115, 4255-4264.

49. Zhang, C.; Lu, P.; Chen, Y. Molecular dynamics simulation of electroosmotic flow in rough nanochannels. International Communications in Heat and Mass Transfer 2014, 59, 101-105.

50. Di Marino, D.; Bonome, E. L.; Tramontano, A.; Chinappi, M. All-Atom Molecular Dynamics Simulation of Protein Translocation through an $\alpha$-Hemolysin Nanopore. The Journal of Physical Chemistry Letters 2015, 6, 2963-8.

51. Belkin, M.; Aksimentiev, A. Molecular Dynamics Simulation of DNA Capture and Transport in Heated Nanopores. ACS Applied Materials \& Interfaces 2016, 8, 12599-608. 
52. Schirmer, T.; Phale, P. S. Brownian dynamics simulation of ion flow through porin channels. Journal of Molecular Biology 1999, 294, 1159-67.

53. Im, W.; Roux, B. Ion Permeation and Selectivity of OmpF Porin: A Theoretical Study Based on Molecular Dynamics, Brownian Dynamics, and Continuum Electrodiffusion Theory. Journal of Molecular Biology 2002, 322, 851-869.

54. Noskov, S. Y.; Im, W.; Roux, B. Ion permeation through the alpha-hemolysin channel: theoretical studies based on Brownian dynamics and Poisson-Nernst-Plank electrodiffusion theory. Biophysical Journal 2004, 87, 2299-309.

55. Millar, C.; Madathil, R.; Beckstein, O.; Sansom, M. S. P.; Roy, S.; Asenov, A. Brownian simulation of charge transport in $\alpha$-Haemolysin. Journal of Computational Electronics 2008, 7, 28-33.

56. Egwolf, B.; Luo, Y.; Walters, D. E.; Roux, B. Ion selectivity of alpha-hemolysin with betacyclodextrin adapter. II. Multi-ion effects studied with grand canonical Monte Carlo/Brownian dynamics simulations. The Journal of Physical Chemistry. B 2010, 114, 2901-9.

57. De Biase, P. M.; Markosyan, S.; Noskov, S. BROMOC suite: Monte Carlo/Brownian dynamics suite for studies of ion permeation and DNA transport in biological and artificial pores with effective potentials. Journal of Computational Chemistry 2015, 36, 264-71.

58. Pederson, E. D.; Barbalas, J.; Drown, B. S.; Culbertson, M. J.; Keranen Burden, L. M.; Kasianowicz, J. J.; Burden, D. L. Proximal Capture Dynamics for a Single Biological Nanopore Sensor. The Journal of Physical Chemistry B 2015, 119, 10448-55.

59. Grochowski, P.; Trylska, J. Continuum molecular electrostatics, salt effects, and counterion binding-a review of the Poisson-Boltzmann theory and its modifications. Biopolymers 2008, 89, 93-113.

60. Baldessari, F.; Santiago, J. G. Electrokinetics in nanochannels: part I. Electric double layer overlap and channel-to-well equilibrium. Journal of Colloid and Interface Science 2008, 325, $526-38$. 
61. Eisenberg, R. Computing the Field in Proteins and Channels. Journal of Membrane Biology 1996, 150, 1-25.

62. Gillespie, D.; Nonner, W.; Eisenberg, R. S. Coupling Poisson-Nernst-Planck and density functional theory to calculate ion flux. Journal of Physics: Condensed Matter 2002, 14, 1212912145.

63. Simakov, N. A.; Kurnikova, M. G. Soft wall ion channel in continuum representation with application to modeling ion currents in $\alpha$-hemolysin. The Journal of Physical Chemistry B 2010, 114, 15180-90.

64. Lu, B.; Hoogerheide, D. P.; Zhao, Q.; Yu, D. Effective driving force applied on DNA inside a solid-state nanopore. Physical Review E 2012, 86, 011921.

65. Corry, B.; Kuyucak, S.; Chung, S. H. Invalidity of continuum theories of electrolytes in nanopores. Chemical Physics Letters 2000, 320, 35-41.

66. Collins, K. D. Why continuum electrostatics theories cannot explain biological structure, polyelectrolytes or ionic strength effects in ion-protein interactions. Biophysical Chemistry 2012, 167, 43-59.

67. Furini, S.; Zerbetto, F.; Cavalcanti, S. Application of the Poisson-Nernst-Planck theory with space-dependent diffusion coefficients to KcsA. Biophysical Journal 2006, 91, 3162-9.

68. Liu, J.-L.; Eisenberg, B. Numerical methods for a Poisson-Nernst-Planck-Fermi model of biological ion channels. Physical Review. E, Statistical, Nonlinear, and Soft Matter Physics 2015, 92, 012711.

69. Aguilella-Arzo, M.; Queralt-Martín, M.; Lopez, M.-L.; Alcaraz, A. Fluctuation-Driven Transport in Biological Nanopores. A 3D Poisson-Nernst-Planck Study. Entropy 2017, 19, 116.

70. Simakov, N. A.; Kurnikova, M. G. Membrane Position Dependency of the $\mathrm{pK}_{\mathrm{a}}$ and Conductivity of the Protein Ion Channel. The Journal of Membrane Biology 2018, 251, 393-404. 
71. Cervera, J.; Schiedt, B.; Ramírez, P. A Poisson/Nernst-Planck model for ionic transport through synthetic conical nanopores. EPL (Europhysics Letters) 2005, 71, 35-41.

72. White, H. S.; Bund, A. Ion current rectification at nanopores in glass membranes. Langmuir 2008, 24, 2212-8.

73. Chaudhry, J. H.; Comer, J.; Aksimentiev, A.; Olson, L. N. A Stabilized Finite Element Method for Modified Poisson-Nernst-Planck Equations to Determine Ion Flow Through a Nanopore. Communications in Computational Physics 2014, 15.

74. Bazant, M. Z.; Kilic, M. S.; Storey, B. D.; Ajdari, A. Towards an understanding of inducedcharge electrokinetics at large applied voltages in concentrated solutions. Advances in Colloid and Interface Science 2009, 152, 48-88.

75. Daiguji, H. Ion transport in nanofluidic channels. Chemical Society Reviews 2010, 39, 901-11.

76. Borukhov, I.; Andelman, D.; Orland, H. Steric Effects in Electrolytes: A Modified PoissonBoltzmann Equation. Physical Review Letters 1997, 79, 435-438.

77. Kilic, M. S.; Bazant, M. Z.; Ajdari, A. Steric effects in the dynamics of electrolytes at large applied voltages. II. Modified Poisson-Nernst-Planck equations. Physical review. E, Statistical, Nonlinear, and Soft Matter Physics 2007, 75, 021503.

78. Lu, B.; Zhou, Y. C. Poisson-Nernst-Planck equations for simulating biomolecular diffusionreaction processes II: size effects on ionic distributions and diffusion-reaction rates. Biophysical Journal 2011, 100, 2475-85.

79. Makarov, V.; Feig, M.; Andrews, B.; Pettitt, B. Diffusion of Solvent around Biomolecular Solutes: A Molecular Dynamics Simulation Study. Biophysical Journal 1998, 75, 150-158.

80. Pronk, S.; Lindahl, E.; Kasson, P. M. Dynamic heterogeneity controls diffusion and viscosity near biological interfaces. Nature Communications 2014, 5, 3034.

81. McMullen, A. J.; Tang, J. X.; Stein, D. Nanopore Measurements of Filamentous Viruses Reveal a Sub-nanometer-Scale Stagnant Fluid Layer. ACS Nano 2017, 11, 11669-11677. 
82. Qiao, R.; Aluru, N. R. Ion concentrations and velocity profiles in nanochannel electroosmotic flows. The Journal of Chemical Physics 2003, 118, 4692.

83. Vo, T. Q.; Kim, B. Transport Phenomena of Water in Molecular Fluidic Channels. Scientific Reports 2016, 6, 33881.

84. Hsu, W.-L.; Harvie, D. J. E.; Davidson, M. R.; Dunstan, D. E.; Hwang, J.; Daiguji, H. Viscoelectric Effects in Nanochannel Electrokinetics. The Journal of Physical Chemistry C 2017, 121, 20517-20523.

85. Ye, H.; Zhang, H.; Zhang, Z.; Zheng, Y. Size and temperature effects on the viscosity of water inside carbon nanotubes. Nanoscale Research Letters 2011, 6, 87.

86. Contreras-Aburto, C.; Nägele, G. A unifying mode-coupling theory for transport properties of electrolyte solutions. I. General scheme and limiting laws. The Journal of Chemical Physics 2013, 139, 134109.

87. Mills, R.; Lobo, V. Self-diffusion in electrolyte solutions: a critical examination of data compiled from the literature; Physical Sciences Data v. 36; Elsevier, 1989.

88. Panopoulos, D. K.; Kaneko, H.; Spiro, M. Transference numbers of sodium chloride in concentrated aqueous solutions and chloride conductances in several concentrated electrolyte solutions. Journal of Solution Chemistry 1986, 15, 243-252.

89. Contreras-Aburto, C.; Nägele, G. A unifying mode-coupling theory for transport properties of electrolyte solutions. II. Results for equal-sized ions electrolytes. The Journal of Chemical Physics 2013, 139, 134110.

90. Baldessari, F.; Santiago, J. G. Electrokinetics in nanochannels: part II. Mobility dependence on ion density and ionic current measurements. Journal of Colloid and Interface Science 2008, $325,539-46$.

91. Burger, M.; Schlake, B.; Wolfram, M.-T. Nonlinear Poisson-Nernst-Planck equations for ion flux through confined geometries. Nonlinearity 2012, 25, 961-990. 
92. Hai-Lang, Z.; Shi-Jun, H. Viscosity and Density of Water + Sodium Chloride + Potassium Chloride Solutions at 298.15 K. Journal of Chemical \& Engineering Data 1996, 41, 516-520.

93. Gavish, N.; Promislow, K. Dependence of the dielectric constant of electrolyte solutions on ionic concentration: A microfield approach. Physical Review E 2016, 94, 012611.

94. Chen, D. A New Poisson-Nernst-Planck Model with Ion-Water Interactions for Charge Transport in Ion Channels. Bulletin of Mathematical Biology 2016, 78, 1703-26.

95. Mueller, M.; Grauschopf, U.; Maier, T.; Glockshuber, R.; Ban, N. The structure of a cytolytic alpha-helical toxin pore reveals its assembly mechanism. Nature 2009, 459, 726-30.

96. Soskine, M.; Biesemans, A.; De Maeyer, M.; Maglia, G. Tuning the size and properties of ClyA nanopores assisted by directed evolution. Journal of the American Chemical Society 2013, 135, 13456-63.

97. Soskine, M.; Biesemans, A.; Maglia, G. Single-Molecule Analyte Recognition with ClyA Nanopores Equipped with Internal Protein Adaptors. Journal of the American Chemical Society 2015, 137, 5793-5797.

98. Biesemans, A.; Soskine, M.; Maglia, G. A Protein Rotaxane Controls the Translocation of Proteins Across a ClyA Nanopore. Nano Letters 2015, 15, 6076-6081.

99. Wloka, C.; Van Meervelt, V.; van Gelder, D.; Danda, N.; Jager, N.; Williams, C. P.; Maglia, G. Label-Free and Real-Time Detection of Protein Ubiquitination with a Biological Nanopore. ACS Nano 2017, 11, 4387-4394.

100. Van Meervelt, V.; Soskine, M.; Singh, S.; Schuurman-Wolters, G. K.; Wijma, H. J.; Poolman, B.; Maglia, G. Real-Time Conformational Changes and Controlled Orientation of Native Proteins Inside a Protein Nanoreactor. Journal of the American Chemical Society 2017, 139, 18640-18646.

101. Franceschini, L.; Brouns, T.; Willems, K.; Carlon, E.; Maglia, G. DNA Translocation through Nanopores at Physiological Ionic Strengths Requires Precise Nanoscale Engineering. ACS Nano 2016, 10, 8394-402. 
102. Humphrey, W.; Dalke, A.; Schulten, K. VMD: Visual molecular dynamics. Journal of Molecular Graphics 1996, 14, 33-38.

103. Stone, J. An Efficient Library for Parallel Ray Tracing and Animation. M.Sc. thesis, Computer Science Department, University of Missouri-Rolla, 1998.

104. Li, L.; Li, C.; Zhang, Z.; Alexov, E. On the Dielectric "Constant" of Proteins: Smooth Dielectric Function for Macromolecular Modeling and Its Implementation in DelPhi. Journal of Chemical Theory and Computation 2013, 9, 2126-2136.

105. Gramse, G.; Dols-Perez, A.; Edwards, M. A.; Fumagalli, L.; Gomila, G. Nanoscale measurement of the dielectric constant of supported lipid bilayers in aqueous solutions with electrostatic force microscopy. Biophysical Journal 2013, 104, 1257-62.

106. Bianchi, H.; Corti, H. R.; Fernández-Prini, R. The conductivity of concentrated aqueous mixtures of $\mathrm{NaCl}$ and $\mathrm{MgCl} 2$ at $25^{\circ} \mathrm{C}$. Journal of Solution Chemistry 1989, 18, 485-491.

107. Currie, D. J.; Gordon, A. R. TRANSFERENCE NUMBERS FOR CONCENTRATED AQUEOUS SODIUM CHLORIDE SOLUTIONS, AND THE IONIC CONDUCTANCES FOR POTASSIUM AND SODIUM CHLORIDES. The Journal of Physical Chemistry 1960, $64,1751-1753$.

108. Goldsack, D. E.; Franchetto, R.; Franchetto, A. A. Solvation effects on the conductivity of concentrated electrolyte solutions. Canadian Journal of Chemistry 1976, 54, 2953-2966.

109. Della Monica, M.; Petrella, G.; Sacco, A.; Bufo, S. Transference numbers in concentrated sodium chloride solutions. Electrochimica Acta 1979, 24, 1013-1017.

110. Renkin, E. M. Filtration, diffusion, and molecular sieving through porous cellulose membranes. The Journal of General Physiology 1954, 38, 225-43.

111. Deen, W. M. Hindered transport of large molecules in liquid-filled pores. AIChE Journal 1987, 33, 1409-1425. 
112. Dechadilok, P.; Deen, W. M. Hindrance Factors for Diffusion and Convection in Pores. Industrial \& Engineering Chemistry Research 2006, 45, 6953-6959.

113. Kannam, S. K.; Downton, M. T. Translational diffusion of proteins in nanochannels. The Journal of Chemical Physics 2017, 146, 054108.

114. Anderson, J. L.; Quinn, J. A. Ionic mobility in microcapillaries. A test for anomalous water structures. Journal of the Chemical Society, Faraday Transactions 1: Physical Chemistry in Condensed Phases 1972, 68, 744.

115. Axelsson, O.; He, X.; Neytcheva, M. Numerical Solution of the Time-Dependent NavierStokes Eequation for Variable Density-Variable Viscosity. Part I. Mathematical Modelling and Analysis 2015, 20, 232-260.

116. Kowalczyk, S. W.; Grosberg, A. Y.; Rabin, Y.; Dekker, C. Modeling the Conductance and DNA Blockade of Solid-State Nanopores. Nanotechnology 2011, 22, 315101.

117. Constantin, D.; Siwy, Z. S. Poisson-Nernst-Planck model of ion current rectification through a nanofluidic diode. Physical review. E, Statistical, Nonlinear, and Soft Matter Physics 2007, $76,041202$.

118. Uematsu, Y.; Netz, R. R.; Bocquet, L.; Bonthuis, D. J. Crossover of the Power-Law Exponent for Carbon Nanotube Conductivity as a Function of Salinity. The Journal of Physical Chemistry. B 2018, 122, 2992-2997.

119. Duan, C.; Majumdar, A. Anomalous ion transport in 2-nm hydrophilic nanochannels. Nature Nanotechnology 2010, 5, 848-52.

120. Basdevant, N.; Dessaux, D.; Ramirez, R. Ionic transport through a protein nanopore: a Coarse-Grained Molecular Dynamics Study. Scientific Reports 2019, 9.

121. Thompson, A. P. Nonequilibrium molecular dynamics simulation of electro-osmotic flow in a charged nanopore. The Journal of Chemical Physics 2003, 119, 7503-7511. 
122. Firnkes, M.; Pedone, D.; Knezevic, J.; Döblinger, M.; Rant, U. Electrically Facilitated Translocations of Proteins through Silicon Nitride Nanopores: Conjoint and Competitive Action of Diffusion, Electrophoresis, and Electroosmosis. Nano Letters 2010, 10, 2162-2167.

123. Li, S.; Cao, C.; Yang, J.; Long, Y.-T. Detection of Peptides with Different Charges and Lengths by Using the Aerolysin Nanopore. ChemElectroChem 2018, 6, 126-129.

124. Huang, G.; Voet, A.; Maglia, G. FraC nanopores with adjustable diameter identify the mass of opposite-charge peptides with 44 dalton resolution. Nature Communications 2019, 10.

125. Willems, K.; Ruić, D.; Biesemans, A.; Galenkamp, N. S.; Dorpe, P. V.; Maglia, G. Engineering and Modeling the Electrophoretic Trapping of a Single Protein Inside a Nanopore. ACS Nano 2019, 13, 9980-9992.

126. Melnikov, D. V.; Hulings, Z. K.; Gracheva, M. E. Electro-osmotic flow through nanopores in thin and ultrathin membranes. Physical Review E 2017, 95.

127. Sali, A.; Blundell, T. L. Comparative protein modelling by satisfaction of spatial restraints. Journal of Molecular Biology 1993, 234, 779-815.

128. Shanno, D. F.; Phua, K. H. Remark on "Algorithm 500: Minimization of Unconstrained Multivariate Functions [E4]". ACM Transactions on Mathematical Software 1980, 6, 618622.

129. Jo, S.; Kim, T.; Iyer, V. G.; Im, W. CHARMM-GUI: a web-based graphical user interface for CHARMM. Journal of Computational Chemistry 2008, 29, 1859-65.

130. Lee, J.; Cheng, X.; Swails, J. M.; Yeom, M. S.; Eastman, P. K.; Lemkul, J. A.; Wei, S.; Buckner, J.; Jeong, J. C.; Qi, Y.; Jo, S.; Pande, V. S.; Case, D. A.; Brooks, C. L.; MacKerell, A. D.; Klauda, J. B.; Im, W. CHARMM-GUI Input Generator for NAMD, GROMACS, AMBER, OpenMM, and CHARMM/OpenMM Simulations Using the CHARMM36 Additive Force Field. Journal of Chemical Theory and Computation 2016, 12, 405-13. 
131. Phillips, J. C.; Braun, R.; Wang, W.; Gumbart, J.; Tajkhorshid, E.; Villa, E.; Chipot, C.; Skeel, R. D.; Kalé, L.; Schulten, K. Scalable molecular dynamics with NAMD. Journal of Computational Chemistry 2005, 26, 1781-802.

132. Wu, E. L.; Cheng, X.; Jo, S.; Rui, H.; Song, K. C.; Dávila-Contreras, E. M.; Qi, Y.; Lee, J.; Monje-Galvan, V.; Venable, R. M.; Klauda, J. B.; Im, W. CHARMM-GUI Membrane Builder toward realistic biological membrane simulations. Journal of Computational Chemistry 2014, 35, 1997-2004.

133. Best, R. B.; Zhu, X.; Shim, J.; Lopes, P. E. M.; Mittal, J.; Feig, M.; Mackerell, A. D. Optimization of the additive CHARMM all-atom protein force field targeting improved sampling of the backbone $\varphi, \psi$ and side-chain $\chi(1)$ and $\chi(2)$ dihedral angles. Journal of Chemical Theory and Computation 2012, 8, 3257-3273.

134. Olsson, M. H. M.; Søndergaard, C. R.; Rostkowski, M.; Jensen, J. H. PROPKA3: Consistent Treatment of Internal and Surface Residues in Empirical pKa Predictions. Journal of Chemical Theory and Computation 2011, 7, 525-37.

135. Jurrus, E.; Engel, D.; Star, K.; Monson, K.; Brandi, J.; Felberg, L. E.; Brookes, D. H.; Wilson, L.; Chen, J.; Liles, K.; Chun, M.; Li, P.; Gohara, D. W.; Dolinsky, T.; Konecny, R.; Koes, D. R.; Nielsen, J. E.; Head-Gordon, T.; Geng, W.; Krasny, R.; Wei, G.-W.; Holst, M. J.; McCammon, J. A.; Baker, N. A. Improvements to the APBS biomolecular solvation software suite. Protein Science 2018, 27, 112-128. 
bioRxiv preprint doi: https://doi.org/10.1101/2020.01.08.897819; this version posted January 9, 2020. The copyright holder for this preprint (which was not certified by peer review) is the author/funder, who has granted bioRxiv a license to display the preprint in perpetuity. It is made available under aCC-BY 4.0 International license.

\section{Graphical TOC Entry}

